TRANSACTIONS OF THE

AMERICAN MATHEMATICAL SOCIETY

Volume 358, Number 1, Pages 87-114

S 0002-9947(05)04068-7

Article electronically published on August 25, 2005

\title{
BOCHNER-WEITZENBÖCK FORMULAS AND CURVATURE ACTIONS ON RIEMANNIAN MANIFOLDS
}

\author{
YASUSHI HOMMA
}

\begin{abstract}
Gradients are natural first order differential operators depending on Riemannian metrics. The principal symbols of them are related to the enveloping algebra and higher Casimir elements. We give formulas in the enveloping algebra that induce not only identities for higher Casimir elements but also all Bochner-Weitzenböck formulas for gradients. As applications, we give some vanishing theorems.
\end{abstract}

\section{INTRODUCTION}

The Dirac operator is an important tool in a wide range of mathematics and physics. The principal symbols of the Dirac operator are known as the Clifford multiplications and constitute the Clifford algebra. The algebra not only gives features of the Dirac operator but also has importance by itself [19]. We can generalize the Dirac operator under the condition that operators are first order and conformally covariant 10, 22. The generalized operators are called gradients or Stein-Weiss operators and include basic operators in Riemannian and spin geometry: the exterior derivative, the interior derivative, the conformal Killing operator, the twistor operator and the Rarita-Schwinger operator. From recent research by T. Branson et al., we know various properties and applications of gradients, which give a new direction in geometry and analysis.

Let $(M, g)$ be an $n$-dimensional oriented Riemannian manifold and let $\mathbf{S O}(M)$ be the bundle of oriented orthonormal frames with structure group $\mathrm{SO}(n)$. An irreducible unitary $\mathrm{SO}(n)$-module $V_{\rho}$ with highest weight $\rho$ gives an associated vector bundle $\mathbf{S}_{\rho}:=\mathbf{S O}(M) \times_{\pi_{\rho}} V_{\rho}$ on $M$. On this vector bundle, we have a covariant derivative $\nabla$ induced from the Levi-Civita connection. We decompose the target bundle of $\nabla$ as

$$
\mathbf{S}_{\rho} \otimes T_{\mathbb{C}}^{*}(M)=\bigoplus_{1 \leq i \leq N} \mathbf{S}_{\lambda_{i}}
$$

Then the derivative $\nabla$ splits along this decomposition, and each summand is a first order differential operator,

$$
D_{\lambda_{i}}^{\rho}: \Gamma\left(M, \mathbf{S}_{\rho}\right) \stackrel{\nabla}{\longrightarrow} \Gamma\left(M, \mathbf{S}_{\rho} \otimes T_{\mathbb{C}}^{*}(M)\right) \stackrel{\text { projection }}{\longrightarrow} \Gamma\left(M, \mathbf{S}_{\lambda_{i}}\right) .
$$

Received by the editors July 3, 2003.

2000 Mathematics Subject Classification. Primary 53B20, 58J60, 17B35.

Key words and phrases. Invariant operators, Bochner-Weitzenböck formulas, $\mathrm{SO}(n)$-modules, Casimir elements.

The author was supported by the Grant-in-Aid for JSPS Fellows for Young Scientists. 
We call this operator gradient. In [3, T. Branson discusses ellipticities of gradients by using the spectral resolution on the standard sphere. As a corollary, he shows that suitable linear combinations of gradients are bundle endomorphisms depending on the Riemannian curvature,

$$
\sum_{1 \leq i \leq N} b_{\lambda_{i}}\left(D_{\lambda_{i}}^{\rho}\right)^{*} D_{\lambda_{i}}^{\rho}=\text { curvature action. }
$$

These formulas are called optimal Bochner-Weitzenböck formulas. The vector $\left(b_{\lambda_{1}}, \cdots, b_{\lambda_{N}}\right)$ needs to be a solution to a certain system of linear equations (see [3, Theorem 5.10]). Since the solutions constitute an [N/2]-dimensional subspace in $\mathbb{R}^{N}$, there are $[N / 2]$ independent optimal Bochner-Weitzenböck formulas. This result induces vanishing theorems and eigenvalue estimates in Riemannian and spin geometry [6], 7], 8]. Furthermore, his approach of [3] is used to solve the Kato constant problem [5]. But it would be complicated to calculate the coefficients $\left\{b_{\lambda_{i}}\right\}_{i}$ and curvature actions in (1.1). On the other hand, D. Calderbank, P. Gauduchon and M. Herzlich also discuss the Kato constant problem [9. Their key observation is that the principal symbols of gradients are related to the enveloping algebra of $\mathfrak{s o}(n)$, especially higher Casimir elements. This suggests that we can reconstruct Branson's optimal Bochner-Weitzenböck formulas in terms of the enveloping algebra. In this paper, we develop the observation of 9 further, and connect higher Casimir elements to Bochner-Weitzenböck formulas. Consequently, we obtain a universal and direct construction of the coefficients $\left\{b_{\lambda_{i}}\right\}_{i}$ and curvature actions in (1.1), which makes it possible to give various vanishing theorems and eigenvalue estimates.

In Section 2, we give a short review to representations of $\mathfrak{s o}(n)$. In Section 3 , after we discuss the enveloping algebra and Casimir elements of $\mathfrak{s o}(n)$, we give the universal Bochner-Weitzenböck formulas (Theorem 3.4). The formulas induce some identities for higher Casimir elements (Corollary [3.5). In Section 4 we discuss the principal symbols of gradients called Clifford homomorphisms. We relate them with the enveloping algebra by using conformal weights. From the universal Bochner-Weitzenböck formulas, we have identities for Clifford homomorphisms corresponding to (1.1) on the symbol level. We also compute eigenvalues of Casimir elements. In Section [5 we define gradients and give some fundamental properties of them. In Section [6 we introduce curvature endomorphisms corresponding to curvature actions in (1.1). An interesting observation is that the curvature endomorphism associated to the Pfaffian element depends only on the conformal Weyl tensor and the scalar curvature (Proposition 6.5). In Section 7, we give BochnerWeitzenböck formulas for gradients (Theorem 7.1) and show that our formulas give an answer to the problem of finding out $\left\{b_{\lambda_{i}}\right\}_{i}$ in (1.1). We also discuss a relation between our Bochner-Weitzenböck formulas and Branson's conformally covariant operators in 4. In Section 8 after mentioning gradients on spinors and differential forms, we apply our formulas to vanishing theorems for other gradients. In the last section, we discuss the four-dimensional case. Because of $\Lambda^{2}=\Lambda_{+}^{2} \oplus \Lambda_{-}^{2}$, we can calculate curvature endomorphisms more precisely and obtain vanishing theorems for higher tensor-spinor fields.

An outline of some results has been presented in a short paper [16] by the author. We discuss the details and develop them in the present paper. New results and many examples are included. 


\section{Representations of $\mathrm{SO}(n)$ and $\operatorname{Spin}(n)$}

In this section, we give a short review to representation theory of the special orthogonal group $\mathrm{SO}(n)$ or the spin group $\operatorname{Spin}(n)$ [18, 24]. Let $\mathbb{R}^{n}$ be the $n$ dimensional Euclidean space with inner product $\langle\cdot, \cdot\rangle$ and standard basis $\left\{e_{i}\right\}_{i=1}^{n}$. Associating $\xi \wedge \eta$ with the skew-symmetric endomorphism

$$
(\xi \wedge \eta)(u)=\langle\xi, u\rangle \eta-\langle\eta, u\rangle \xi \text { for } u \in \mathbb{R}^{n},
$$

we identify $\Lambda^{2}\left(\mathbb{R}^{n}\right)$ with the Lie algebra $\mathfrak{s o}(n)$ of $\operatorname{SO}(n)$ or $\operatorname{Spin}(n)$. Set $e_{i j}:=e_{i} \wedge e_{j}$, and we know that $\left\{e_{i j}\right\}_{1 \leq i, j \leq n}$ satisfy

$$
\begin{aligned}
e_{i j} & =-e_{j i}, \\
{\left[e_{k l}, e_{i j}\right] } & =\delta_{k i} e_{l j}+\delta_{k j} e_{i l}-\delta_{i l} e_{k j}-\delta_{l j} e_{i k},
\end{aligned}
$$

and $\left\{e_{i j} \mid 1 \leq i<j \leq n\right\}$ constitute a basis of $\mathfrak{s o}(n)$.

We choose a Cartan subalgebra $\mathfrak{h}$ of $\mathfrak{s o}(n)$ with basis $\left\{e_{2 i-1,2 i} \mid 1 \leq i \leq m=\right.$ $[n / 2]\}$ and fix a basis $\left\{\mu_{i}\right\}_{1 \leq i \leq m}$ of $(\sqrt{-1} \mathfrak{h})^{*}$ such that $\mu_{i}\left(-\sqrt{-1} e_{2 j-1,2 j}\right)=\delta_{i j}$. Normalizing the Killing form on $\mathfrak{s o}(n)$, we have a positive definite inner product on $(\sqrt{-1} \mathfrak{h})^{*}$ such that $\left\langle\mu_{i}, \mu_{j}\right\rangle=\delta_{i j}$. Thus we regard $(\sqrt{-1} \mathfrak{h}) *$ as the $m$-dimensional Euclidean space and denote $\mu_{i}$ by

$$
\mu_{i}=(\underbrace{0, \cdots, 0}_{i-1}, 1, \underbrace{0, \cdots, 0}_{m-i}) .
$$

We consider a finite-dimensional irreducible unitary representation $(\pi, V)$ of $\mathrm{SO}(n)$ or $\operatorname{Spin}(n)$, and decompose the representation space $V$ into simultaneous eigenspaces with respect to $\sqrt{-1} \mathfrak{h}$. Each eigenvalue $\nu$ called weight is an integral or half-integral linear combination of $\left\{\mu_{i}\right\}_{i}$, namely, $\nu=\sum \nu^{i} \mu_{i}=\left(\nu^{1}, \cdots, \nu^{m}\right)$ in $\mathbb{Z}^{m} \cup(\mathbb{Z}+1 / 2)^{m}$. Ordering the weights lexicographically, we have the highest weight $\rho=\left(\rho^{1}, \cdots, \rho^{m}\right)$ for $(\pi, V)$ with multiplicity one. This highest weight satisfies the dominant condition

$$
\begin{aligned}
\rho^{1} \geq \rho^{2} \geq \cdots \geq \rho^{m-1} \geq\left|\rho^{m}\right|, \quad \text { for } n=2 m, \\
\rho^{1} \geq \rho^{2} \geq \cdots \geq \rho^{m-1} \geq \rho^{m} \geq 0, \quad \text { for } n=2 m+1 .
\end{aligned}
$$

Conversely, for a dominant weight $\rho$ in $\mathbb{Z}^{m} \cup(\mathbb{Z}+1 / 2)^{m}$, we can construct a unique irreducible unitary representation with highest weight $\rho$ up to equivalence. Therefore we denote by $\left(\pi_{\rho}, V_{\rho}\right)$ an irreducible representation with highest weight $\rho$ of $\operatorname{SO}(n), \operatorname{Spin}(n)$ or $\mathfrak{s o}(n)$. Note that if $\rho$ is half-integral, then $\left(\pi_{\rho}, V_{\rho}\right)$ does not factor through a representation of $\mathrm{SO}(n)$. When writing dominant weights, we denote by $k_{j}$ a string of $k$ with length $j$ and sometimes omit a terminal string of zeros. For example, the highest weight of the spinor representation is

$$
\left((1 / 2)_{m-1}, \pm 1 / 2\right)=(\underbrace{1 / 2,1 / 2, \cdots, 1 / 2}_{m-1}, \pm 1 / 2),
$$

and the one of the representation on $\Lambda^{p}\left(\mathbb{R}^{n}\right) \otimes \mathbb{C}$ is

$$
\left(1_{p}\right)=(\underbrace{1, \cdots, 1}_{p}, \underbrace{0, \cdots, 0}_{m-p}) .
$$




\section{Enveloping algebra And higher Casimir elements}

Let $\mathfrak{s o}(n, \mathbb{C})$ be the complexification of $\mathfrak{s o}(n)$ and let $U(\mathfrak{s o}(n, \mathbb{C}))$ be its enveloping algebra. The enveloping algebra is the quotient algebra of the tensor algebra of $\mathfrak{s o}(n, \mathbb{C})$ by two-sided ideal generated by all $(X \otimes Y-Y \otimes X-[X, Y])$ for $X, Y$ in $\mathfrak{s o}(n, \mathbb{C})$. Each representation $(\pi, V)$ of $\mathfrak{s o}(n)$ is lifted naturally to a representation of the enveloping algebra denoted by the same notation $(\pi, V)$.

The center $\mathfrak{Z}$ of $U(\mathfrak{s o}(n, \mathbb{C}))$ is characterized as the invariant subalgebra in $U(\mathfrak{s o}(n, \mathbb{C}))$ under the adjoint action of $\mathrm{SO}(n)$. We call elements in $\mathfrak{Z}$ Casimir elements. From Schur's lemma, we know that every Casimir element is a constant on irreducible $\mathfrak{s o}(n)$-module.

We shall construct an algebraic basis of $\mathfrak{Z}$ [20, 21], 24]. First, we define the usual Casimir element by $c_{2}:=\sum_{i, j} e_{i j} e_{j i}$. It is known that the eigenvalue of $c_{2}$ on irreducible $\mathfrak{s o}(n)$-module $V_{\rho}$ is

$$
\pi_{\rho}\left(c_{2}\right)=2\langle\delta+\rho, \delta+\rho\rangle-2\langle\delta, \delta\rangle=2\langle\rho, \rho\rangle+4\langle\rho, \delta\rangle,
$$

where $\delta$ is half the sum of the positive roots

$$
\delta= \begin{cases}(m-1, m-2, \cdots, 1,0) & \text { for } n=2 m, \\ (m-1 / 2, m-3 / 2, \cdots, 3 / 2,1 / 2) & \text { for } n=2 m+1 .\end{cases}
$$

For example, on the natural representation $\left(\pi_{\mu_{1}}, V_{\mu_{1}}\right)=\left(\pi_{\mu_{1}}, \mathbb{C}^{n}\right)$, we have $\pi_{\mu_{1}}\left(c_{2}\right)$ $=2(n-1)$.

Next we construct higher Casimir elements. For each non-negative integer $q$, we define an element $e_{i j}^{q}$ in $U(\mathfrak{s o}(n, \mathbb{C}))$ by

$$
e_{i j}^{q}:= \begin{cases}\sum_{1 \leq i_{1}, i_{2}, \cdots, i_{q-1} \leq n} e_{i i_{1}} e_{i_{1} i_{2}} \cdots e_{i_{q-1} j}, & q \geq 1, \\ \delta_{i j}, & q=0 .\end{cases}
$$

This $e_{i j}^{q}$ behaves like $e_{i j}$ under the adjoint action of $\mathfrak{s o}(n)$ as follows.

Lemma 3.1. The elements $\left\{e_{i j}^{q} \mid q \in \mathbb{Z}_{\geq 0}, i, j=1, \cdots, n\right\}$ satisfy

$$
\begin{aligned}
{\left[e_{k l}, e_{i j}^{q}\right]=} & \delta_{k i} e_{l j}^{q}+\delta_{k j} e_{i l}^{q}-\delta_{i l} e_{k j}^{q}-\delta_{l j} e_{i k}^{q}, \\
& \sum_{1 \leq k \leq n} e_{i k}^{p} e_{k j}^{q}=e_{i j}^{p+q} .
\end{aligned}
$$

Proof. We calculate the adjoint action of $\mathfrak{s o}(n)$ on $e_{i j}^{q}$. From (2.1), we have

$$
\begin{aligned}
{\left[e_{k l}, e_{i j}^{q}\right] } & =\sum_{i_{1}, \cdots, i_{q-1}}\left[e_{k l}, e_{i i_{1}}\right] e_{i_{1} i_{2}} \cdots e_{i_{q-1} j}+\cdots+\sum_{i_{1}, \cdots, i_{q-1}} e_{i i_{1}} e_{i_{1} i_{2}} \cdots\left[e_{k l}, e_{i_{q-1} j}\right] \\
& =\delta_{k i} e_{l j}^{q}+\delta_{k j} e_{i l}^{q}-\delta_{i l} e_{k j}^{q}-\delta_{l j} e_{i k}^{q} .
\end{aligned}
$$

The equation (3.4) is clear from the definition (3.2).

The equation (3.3) implies that the trace $c_{q}:=\sum_{i} e_{i i}^{q}$ is an invariant element. Thus we have higher Casimir elements $\left\{c_{q}\right\}_{q \geq 0}$. Note that $c_{0}$ is equal to $n$ and $c_{1}$ is zero. The eigenvalues of $c_{q}$ on irreducible $\mathfrak{s o}(n)$-modules are calculated in Section 4 ,

In the case of $n=2 m+1$, Casimir elements $\left\{c_{q}\right\}_{q}$ generate the center $\mathfrak{Z}$ algebraically. On the other hand, in the case of $n=2 m$, we need another Casimir 
element to generate $\mathfrak{Z}$. We define pf in $\mathfrak{Z}$ by

$$
\text { pf }:=\frac{1}{(\sqrt{-1})^{m} 2^{m} m !} \sum_{\sigma \in \mathfrak{S}_{2 m}} \operatorname{sign}(\sigma) e_{\sigma(1) \sigma(2)} e_{\sigma(3) \sigma(4)} \cdots e_{\sigma(2 m-1) \sigma(2 m)},
$$

where $\mathfrak{S}_{2 m}$ is the permutation group of $\{1, \cdots, 2 m\}$. We call the Casimir element pf the Pfaffian element. The following facts on these Casimir elements are known. (See [24, pp. 368-373].)

Proposition 3.2. (1) In the case of $n=2 m,\left\{c_{2}, c_{4}, \cdots, c_{2 m-2}\right.$,pf $\}$ generate 3 algebraically. The eigenvalue of $\mathrm{pf}$ on an irreducible $\mathfrak{s o}(2 m)$-module $V_{\rho}$ is

$$
\pi_{\rho}(\mathrm{pf})=\left(\rho^{1}+m-1\right)\left(\rho^{2}+m-2\right) \cdots\left(\rho^{m-1}+1\right) \rho^{m} .
$$

(2) In the case of $n=2 m+1,\left\{c_{2}, c_{4}, \cdots, c_{2 m}\right\}$ generate $\mathfrak{Z}$ algebraically.

From the above proposition, we have the problem of how to realize $c_{2 q+1}$ as a polynomial in $\left\{c_{2 p}\right\}_{p}$. To answer it, we search to find how $e_{i j}^{q}$ is related to $e_{j i}^{q}$. From (3.3), we have

$$
e_{i j}^{q+1}=(1-n) e_{i j}^{q}+\delta_{j i} c_{q}-e_{j i}^{q}-\sum_{k} e_{k j}^{q} e_{k i} .
$$

This equation implies that $e_{i j}^{q}$ is a linear combination of $\left\{e_{j i}^{p}\right\}_{p=0}^{q}$ as

$$
e_{i j}^{q}=\sum_{p=0}^{q} a_{q, p} e_{j i}^{p} .
$$

Here $\left\{a_{q, p}\right\}_{q \geq p \geq 0}$ in $\mathfrak{Z}$ are given by a recursion formula. Since the recursion formula is complicated, we translate $e_{i j}^{q}$ to another element. We define $\hat{e}_{i j}$ by

$$
\hat{e}_{i j}:=e_{i j}+\frac{n-1}{2} \delta_{i j},
$$

and $\hat{e}_{i j}^{q}$ by

$$
\hat{e}_{i j}^{q}:= \begin{cases}\sum_{1 \leq i_{1}, i_{2}, \cdots, i_{q-1} \leq n} \hat{e}_{i i_{1}} \hat{e}_{i_{1} i_{2}} \cdots \hat{e}_{i_{q-1} j}, & q \geq 1, \\ \delta_{i j}, & q=0 .\end{cases}
$$

Note that $\hat{e}_{i j}^{q}$ is related to $e_{i j}^{q}$ as

$$
\hat{e}_{i j}^{q}=\sum_{p=0}^{q}\left(\begin{array}{l}
q \\
p
\end{array}\right)\left(\frac{n-1}{2}\right)^{q-p} e_{i j}^{p} .
$$

We also define the translated Casimir element $\hat{c}_{q}$ by $\hat{c}_{q}:=\sum_{i} \hat{e}_{i i}^{q}$.

Lemma 3.3. The translated elements $\left\{\hat{e}_{i j}^{q} \mid q \in \mathbb{Z}_{\geq 0}, i, j=1, \cdots, n\right\}$ satisfy

$$
\begin{gathered}
{\left[\hat{e}_{k l}, \hat{e}_{i j}^{q}\right]=\delta_{k i} \hat{e}_{l j}^{q}+\delta_{k j} \hat{e}_{i l}^{q}-\delta_{i l} \hat{e}_{k j}^{q}-\delta_{l j} \hat{e}_{i k}^{q},} \\
\sum_{k} \hat{e}_{i k}^{p} \hat{e}_{k j}^{q}=\hat{e}_{i j}^{p+q}, \\
\hat{e}_{i j}=-\hat{e}_{j i}+(n-1) \delta_{i j} .
\end{gathered}
$$

We particularly obtain a key relation

$$
\hat{e}_{i j}^{q+1}=\delta_{j i} \hat{c}_{q}-\hat{e}_{j i}^{q}-\sum_{k} \hat{e}_{k j}^{q} \hat{e}_{k i}
$$


Proof. We can prove (3.7)-(3.9) in the same way as Lemma 3.1. It follows from (3.7) - (3.9) that

$$
\begin{aligned}
\hat{e}_{i j}^{q+1} & =\sum_{k}\left(\left[\hat{e}_{i k}, \hat{e}_{k j}^{q}\right]+\hat{e}_{k j}^{q} \hat{e}_{i k}\right) \\
& =\sum_{k}\left\{\left(\delta_{i k} \hat{e}_{k j}^{q}+\delta_{i j} \hat{e}_{k k}^{q}-\delta_{k k} \hat{e}_{i j}^{q}-\delta_{k j} \hat{e}_{k i}^{q}\right)+\hat{e}_{k j}^{q}\left(-\hat{e}_{k i}+(n-1) \delta_{i k}\right)\right\} \\
& =\hat{e}_{i j}^{q}+\delta_{i j} \hat{c}_{q}-n \hat{e}_{i j}^{q}-\hat{e}_{j i}^{q}-\sum_{k} \hat{e}_{k j}^{q} \hat{e}_{k i}+(n-1) \hat{e}_{i j}^{q} \\
& =\delta_{i j} \hat{c}_{q}-\hat{e}_{j i}^{q}-\sum_{k} \hat{e}_{k j}^{q} \hat{e}_{k i} .
\end{aligned}
$$

Thus we obtain (3.10).

By using the above lemma, we connect $\hat{e}_{i j}^{q}$ with $\hat{e}_{j i}^{q}$.

Theorem 3.4. The translated element $\hat{e}_{i j}^{q}$ is a linear combination of $\left\{\hat{e}_{j i}^{p}\right\}_{p=0}^{q}$ whose coefficients are Casimir elements,

$$
\hat{e}_{i j}^{q}=(-1)^{q} \hat{e}_{j i}^{q}-\frac{1-(-1)^{q}}{2} \hat{e}_{j i}^{q-1}+\sum_{p=0}^{q-1}(-1)^{p} \hat{c}_{q-1-p} \hat{e}_{j i}^{p} .
$$

Then we have

$$
\begin{gathered}
\hat{e}_{i j}^{2 q}=\hat{e}_{j i}^{2 q}+\sum_{p=0}^{2 q-1}(-1)^{p} \hat{c}_{2 q-1-p} \hat{e}_{j i}^{p}, \\
\hat{e}_{i j}^{2 q+1}=-\hat{e}_{j i}^{2 q+1}-\hat{e}_{j i}^{2 q}+\sum_{p=0}^{2 q}(-1)^{p} \hat{c}_{2 q-p} \hat{e}_{j i}^{p} .
\end{gathered}
$$

Proof. Setting $\hat{e}_{i j}^{q}=\sum_{p=0}^{q} \hat{a}_{q, p} \hat{e}_{j i}^{p}$, we shall produce a recursion formula of $\left\{\hat{a}_{q, p}\right\}_{q \geq p \geq 0}$ in $\mathfrak{Z}$. It follows from (3.10) that

$$
\begin{aligned}
\hat{e}_{i j}^{q+1} & =\delta_{j i} \hat{c}_{q}-\hat{e}_{j i}^{q}-\sum_{k} \hat{e}_{k j}^{q} \hat{e}_{k i} \\
& =\delta_{j i} \hat{c}_{q}-\hat{e}_{j i}^{q}-\sum_{k} \sum_{p} \hat{a}_{q, p} \hat{e}_{j k}^{p} \hat{e}_{k i} \\
& =\delta_{j i} \hat{c}_{q}-\hat{e}_{j i}^{q}-\sum_{p=0}^{q} \hat{a}_{q, p} \hat{e}_{j i}^{p+1} \\
& =-\hat{a}_{q, q} \hat{e}_{j i}^{q+1}+\left(-\hat{a}_{q, q-1}-1\right) \hat{e}_{j i}^{q}-\sum_{p=0}^{q-2} \hat{a}_{q, p} \hat{e}_{j i}^{p+1}+\hat{c}_{q} \delta_{j i} \\
& =\sum_{p=0}^{q+1} \hat{a}_{q+1, p} \hat{e}_{j i}^{p} .
\end{aligned}
$$


Then we have a recursion formula for $\left\{\hat{a}_{q, p}\right\}_{q \geq p \geq 0}$,

$$
\hat{a}_{q+1, p}= \begin{cases}-\hat{a}_{q, q}, & p=q+1, \\ -\hat{a}_{q, q-1}-1, & p=q, \\ -\hat{a}_{q, p-1}, & 1 \leq p \leq q-1, \\ \hat{c}_{q}, & p=0 .\end{cases}
$$

Because of $\hat{e}_{i j}^{0}=\hat{e}_{j i}^{0}$ and $\hat{e}_{i j}^{1}=-\hat{e}_{j i}^{1}+(n-1) \hat{e}_{i j}^{0}$, the initial condition of $\hat{a}_{q, p}$ is $\left(\hat{a}_{0,0}, \hat{a}_{1,0}, \hat{a}_{1,1}\right)=(1, n-1,-1)$. Then

$$
\hat{a}_{q+1, p}= \begin{cases}(-1)^{q+1}, & p=q+1, \\ (-1)^{q}(n-1)-\frac{1-(-1)^{q}}{2}=(-1)^{q} \hat{c}_{0}-\frac{1-(-1)^{q+1}}{2}, & p=q, \\ (-1)^{p} \hat{c}_{q-p}, & 0 \leq p \leq q-1 .\end{cases}
$$

Thus we have proved the theorem.

In Section 6, we show that (3.11) induces Bochner-Weitzenböck formulas on Riemannian manifolds. Hence we call (3.11) the universal Bochner-Weitzenböck formula.

Take the trace in (3.13), and we have identities for $\left\{\hat{c}_{q}\right\}_{q \geq 0}$.

Corollary 3.5. The Casimir elements $\left\{\hat{c}_{0}, \hat{c}_{1}, \cdots\right\}$ satisfy

$$
2 \hat{c}_{2 q+1}=-\hat{c}_{2 q}+\sum_{p=0}^{2 q}(-1)^{p} \hat{c}_{2 q-p} \hat{c}_{p}
$$

for $q=0,1, \cdots$.

D. Calderbank pointed out to the author how the formula (3.11) was related with $[9]$. We set

$$
\hat{E}_{i j}^{q}:=-\frac{1+(-1)^{q}}{2} \hat{e}_{i j}^{q}+\sum_{p=0}^{q}(-1)^{p} \hat{c}_{q-p} \hat{e}_{i j}^{p} .
$$

Then the universal Bochner-Weitzenböck formula means $\hat{E}_{i j}^{q}=(-1)^{q} \hat{E}_{j i}^{q}$. This symmetry is the same as that of a formula in [9, Theorem 4.8]. A better point of our formula is that (3.11) is independent of representations. We note that a formula with such symmetry in the enveloping algebra implies a Bochner-Weitzenböck formula. We actually have another formula related to the Pfaffian element independent of (3.11) in the next section.

\section{PRINCIPAL SYMBOLS OF GRADIENTS}

We consider the Clifford multiplication on the spinor space $V_{\Delta}$, where $n$ is odd and $\Delta$ is $\left((1 / 2)_{m}\right)$. The Clifford multiplication is an action of $\xi$ in $\mathbb{R}^{n}$ on $V_{\Delta}$,

$$
V_{\Delta} \ni \phi \mapsto \xi \cdot \phi \in V_{\Delta},
$$

and satisfies the Clifford relation $\xi \cdot \eta \cdot+\eta \cdot \xi \cdot=-2\langle\xi, \eta\rangle$ id. To generalize the Clifford multiplication, we use another definition as follows. We consider the tensor representation $\left(\pi_{\Delta} \otimes \pi_{\mu_{1}}, V_{\Delta} \otimes\left(\mathbb{R}^{n} \otimes \mathbb{C}\right)\right)$ and decompose it into irreducible modules, $V_{\Delta} \otimes \mathbb{C}^{n}=V_{T} \oplus V_{\Delta}$. Here $T$ is $\left(3 / 2,(1 / 2)_{m-1}\right)$. We denote by $\Pi_{\Delta}^{\Delta}$ the orthogonal projection from $V_{\Delta} \otimes \mathbb{C}^{n}$ onto $V_{\Delta}$ and show that $\Pi_{\Delta}^{\Delta}(\phi \otimes \xi)$ is a constant multiple of $\xi \cdot \phi$. Thus the projection $\Pi_{\Delta}^{\Delta}$ gives another definition of the Clifford multiplication. 
We shall generalize the above discussion to other representation spaces. We consider the tensor representation $\left(\pi_{\rho} \otimes \pi_{\mu_{1}}, V_{\rho} \otimes \mathbb{C}^{n}\right)$ and its irreducible decomposition

$$
V_{\rho} \otimes \mathbb{C}^{n}=\bigoplus_{\lambda} V_{\lambda}
$$

The highest weights of irreducible components occur with multiplicity one and are characterized as follows.

Proposition 4.1 ([10, Theorem 3.4]).

(1) When $n=2 m$, or when $n=2 m+1$ and $\rho^{m}=0$, the highest weight of an irreducible component in $V_{\rho} \otimes \mathbb{C}^{n}$ is dominant, and $\rho \pm \mu_{i}$ for $i=1,2, \cdots, m$.

(2) When $n=2 m+1$ and $\rho^{m}>0$, the highest weight is dominant, and $\rho$ or $\rho \pm \mu_{i}$ for $i=1,2, \cdots, m$.

The tensor inner product on $V_{\rho} \otimes \mathbb{C}^{n}$ induces the one on $V_{\lambda}$. Hence each component is orthogonal to others. We denote by $\Pi_{\lambda}^{\rho}$ the orthogonal projection from $V_{\rho} \otimes \mathbb{C}^{n}$ onto $V_{\lambda}$.

Definition 4.2. For $\xi$ in $\mathbb{C}^{n}$, we define a linear mapping $p_{\lambda}^{\rho}(\xi)$ from $V_{\rho}$ to $V_{\lambda}$ by

$$
\mathbb{C}^{n} \times V_{\rho} \ni(\xi, \phi) \mapsto p_{\lambda}^{\rho}(\xi) \phi:=\Pi_{\lambda}^{\rho}(\phi \otimes \xi) \in V_{\lambda} .
$$

We denote by $p_{\lambda}^{\rho}(\xi)^{*}$ the adjoint operator of $p_{\lambda}^{\rho}(\xi)$ with respect to the inner products on $V_{\rho}$ and $V_{\lambda}$. We call these linear mappings $p_{\lambda}^{\rho}(\xi)$ and $p_{\lambda}^{\rho}(\xi)^{*}$ the Clifford homomorphisms associated to $\rho$ and $\lambda$.

We shall investigate properties of Clifford homomorphisms.

Lemma 4.3. The Clifford homomorphism $p_{\lambda}^{\rho}$ satisfies

$$
\sum_{i} p_{\lambda}^{\rho}\left(e_{i}\right) \pi_{\rho}\left(e_{i j}\right)=w(\rho ; \lambda) p_{\lambda}^{\rho}\left(e_{j}\right)
$$

for each $j$. Here, $w(\rho ; \lambda)$ is given by

$$
w(\rho ; \lambda):=\frac{1}{2}(\langle\delta+\lambda, \delta+\lambda\rangle-\langle\delta+\rho, \delta+\rho\rangle-n+1) .
$$

We call this constant $w(\rho ; \lambda)$ the conformal weight associated to $\rho$ and $\lambda$.

Proof. We define an operator $C$ on $V_{\rho} \otimes \mathbb{C}^{n}$ by

$$
C:=\pi_{\rho} \otimes \pi_{\mu_{1}}\left(c_{2}\right)-\pi_{\rho}\left(c_{2}\right) \otimes \mathrm{id}-\mathrm{id} \otimes \pi_{\mu_{1}}\left(c_{2}\right) .
$$

Since $\pi_{\rho} \otimes \pi_{\mu_{1}}\left(c_{2}\right)$ is $\pi_{\lambda}\left(c_{2}\right)$ on an irreducible component $V_{\lambda}$, we show from (3.1) that $C$ is $4 w(\rho ; \lambda)$ id on $V_{\lambda}$. Then we have

$$
C\left(\phi \otimes e_{i}\right)=C\left(\sum_{\lambda} p_{\lambda}^{\rho}\left(e_{i}\right) \phi\right)=\sum_{\lambda} 4 w(\rho ; \lambda) p_{\lambda}^{\rho}\left(e_{i}\right) \phi
$$

for $\phi \otimes e_{i}$ in $V_{\rho} \otimes \mathbb{C}^{n}$. On the other hand, we get

$$
\begin{aligned}
C= & \pi_{\rho} \otimes \pi_{\mu_{1}}\left(c_{2}\right)-\pi_{\rho}\left(c_{2}\right) \otimes \mathrm{id}-\mathrm{id} \otimes \pi_{\mu_{1}}\left(c_{2}\right) \\
= & \sum_{i j}\left(\pi_{\rho}\left(e_{i j}\right) \otimes \mathrm{id}+\mathrm{id} \otimes \pi_{\mu_{1}}\left(e_{i j}\right)\right)\left(\pi_{\rho}\left(e_{j i}\right) \otimes \mathrm{id}+\mathrm{id} \otimes \pi_{\mu_{1}}\left(e_{j i}\right)\right) \\
& \quad-\sum_{i j} \pi_{\rho}\left(e_{i j}\right) \pi_{\rho}\left(e_{j i}\right) \otimes \mathrm{id}-\sum_{i j} \mathrm{id} \otimes \pi_{\mu_{1}}\left(e_{i j}\right) \pi_{\mu_{1}}\left(e_{j i}\right) \\
& =2 \sum_{i j} \pi_{\rho}\left(e_{i j}\right) \otimes \pi_{\mu_{1}}\left(e_{j i}\right) .
\end{aligned}
$$


Then,

$$
\begin{aligned}
C\left(\phi \otimes e_{i}\right) & =2 \sum_{k l} \pi_{\rho}\left(e_{k l}\right) \phi \otimes \pi_{\mu_{1}}\left(e_{l k}\right) e_{i}=2 \sum_{k l} \pi_{\rho}\left(e_{k l}\right) \phi \otimes\left(\delta_{i l} e_{k}-\delta_{k i} e_{l}\right) \\
& =4 \sum_{k} \pi_{\rho}\left(e_{k i}\right) \phi \otimes e_{k}=4 \sum_{\lambda} \sum_{k} p_{\lambda}^{\rho}\left(e_{k}\right) \pi_{\rho}\left(e_{k i}\right) \phi .
\end{aligned}
$$

Therefore we have $\sum_{k} p_{\lambda}^{\rho}\left(e_{k}\right) \pi_{\rho}\left(e_{k i}\right)=w(\rho ; \lambda) p_{\lambda}^{\rho}\left(e_{i}\right)$ for each $\lambda$.

From this lemma, we can relate Clifford homomorphisms to the enveloping algebra. The following proposition has been known in [9] and 21.

Proposition 4.4. The Clifford homomorphisms $\left\{p_{\lambda}^{\rho}\right\}_{\lambda}$ satisfy

$$
\sum_{\lambda} w(\rho ; \lambda)^{q} p_{\lambda}^{\rho}\left(e_{i}\right)^{*} p_{\lambda}^{\rho}\left(e_{j}\right)=\pi_{\rho}\left(e_{i j}^{q}\right)
$$

for $q=0,1, \cdots$, and $i, j=1, \cdots, n$. In particular, we have

$$
\sum_{\lambda} w(\rho ; \lambda)^{q} \sum_{i} p_{\lambda}^{\rho}\left(e_{i}\right)^{*} p_{\lambda}^{\rho}\left(e_{i}\right)=\pi_{\rho}\left(c_{q}\right) .
$$

Proof. For $\phi$ and $\psi$ in $V_{\rho}$, we have

$$
\delta_{i j}\langle\phi, \psi\rangle=\left\langle\phi \otimes e_{i}, \psi \otimes e_{j}\right\rangle=\sum_{\lambda}\left\langle p_{\lambda}^{\rho}\left(e_{i}\right) \phi, p_{\lambda}^{\rho}\left(e_{j}\right) \psi\right\rangle=\left\langle\sum_{\lambda} p_{\lambda}^{\rho}\left(e_{j}\right)^{*} p_{\lambda}^{\rho}\left(e_{i}\right) \phi, \psi\right\rangle .
$$

Then we have proved (4.2) for $q=0$,

$$
\sum_{\lambda} p_{\lambda}^{\rho}\left(e_{j}\right)^{*} p_{\lambda}^{\rho}\left(e_{i}\right)=\delta_{j i}
$$

By using this equation and (4.1), we can prove (4.2) inductively.

We calculate the conformal weights for $\lambda=\rho, \rho \pm \mu_{i}$,

$$
\begin{cases}w\left(\rho ; \rho+\mu_{i}\right)=\rho^{i}+1-i, & i=1, \cdots, m \\ w\left(\rho ; \rho-\mu_{i}\right)=-\rho^{i}-n+i+1, & i=1, \cdots, m \\ w(\rho ; \rho)=-(n-1) / 2 . & \end{cases}
$$

We assume that the number of irreducible components is $N$, that is,

$$
N=\#\left\{\lambda \mid V_{\lambda} \subset V_{\rho} \otimes \mathbb{C}^{n}\right\} .
$$

Arranging them lexicographically as $\lambda_{1}=\rho+\mu_{1}>\lambda_{2}>\cdots>\lambda_{N}$, we show from (4.4) that

$$
w\left(\rho ; \lambda_{1}\right)>w\left(\rho ; \lambda_{2}\right)>\cdots>w\left(\rho ; \lambda_{N}\right)
$$

except for the following case. When $n=2 m$ and $\rho^{m-1}>\rho^{m}=0$, there always exist the highest weights $\lambda_{+}:=\rho+\mu_{m}$ and $\lambda_{-}:=\rho-\mu_{m}$ whose conformal weights coincide. Then we have

$$
w\left(\rho ; \lambda_{1}\right)>w\left(\rho ; \lambda_{2}\right)>\cdots>w\left(\rho ; \lambda_{+}\right)=w\left(\rho ; \lambda_{-}\right)>\cdots>w\left(\rho ; \lambda_{N}\right) .
$$

We call this case the exceptional case. Thus the conformal weights almost differ from each other. It follows from Proposition 4.4 that we obtain

$$
\left(p_{\lambda_{1}}^{\rho}\left(e_{i}\right)^{*} p_{\lambda_{1}}^{\rho}\left(e_{j}\right), \cdots, p_{\lambda_{N}}^{\rho}\left(e_{i}\right)^{*} p_{\lambda_{N}}^{\rho}\left(e_{j}\right)\right) W^{t}=\left(\delta_{i j}, \pi_{\rho}\left(e_{i j}\right), \cdots, \pi_{\rho}\left(e_{i j}^{N-1}\right)\right),
$$


where $W$ is an $N \times N$ Vandermonde matrix

$$
\left(\begin{array}{cccc}
1 & 1 & \cdots & 1 \\
w\left(\rho ; \lambda_{1}\right) & w\left(\rho ; \lambda_{2}\right) & \cdots & w\left(\rho ; \lambda_{N}\right) \\
\cdots & \cdots & \cdots & \cdots \\
w\left(\rho ; \lambda_{1}\right)^{N-1} & w\left(\rho ; \lambda_{2}\right)^{N-1} & \cdots & w\left(\rho ; \lambda_{N}\right)^{N-1}
\end{array}\right) .
$$

Since $W$ is invertible unless it is the exceptional case, the Clifford homomorphism $p_{\lambda}^{\rho}\left(e_{i}\right)^{*} p_{\lambda}^{\rho}\left(e_{j}\right)$ is a linear combination of $\left\{\pi_{\rho}\left(e_{i j}^{q}\right)\right\}_{q}$.

Remark 4.5. It was first realized in 3, pp. 366-368] that the Vandermonde systems of conformal weights were crucial. Moreover, some formulas in [9] are based on the systems.

For the exceptional case, we need the Pfaffian element pf in (3.5) to distinguish $p_{\lambda_{+}}^{\rho}$ from $p_{\lambda_{-}}^{\rho}$.

Definition 4.6. We define an element $\mathrm{pf}_{i j}$ in the enveloping algebra for $i, j=$ $1, \cdots, 2 m$ by

$$
\mathrm{pf}_{i j}:= \begin{cases}\mathrm{pf}, & i=j \\ (-1)^{i+j} \frac{2 m}{(\sqrt{-1})^{m} 2^{m} m !} \sum_{\sigma \in \mathfrak{S}_{2 m}^{i j}} \operatorname{sgn}(\sigma) e_{\sigma(1) \sigma(2)} \cdots e_{\sigma(2 m-1) \sigma(2 m)}, & i<j \\ -\mathrm{pf}_{j i}, & i>j\end{cases}
$$

where $\mathfrak{S}_{2 m}^{i j}$ is the permutation group of $\{1, \cdots, 2 m\} \backslash\{i, j\}$.

Example 4.7 (four-dimensional case). Let $\left\{e_{i}\right\}_{i=1}^{4}$ be an oriented orthonormal basis of $\mathbb{R}^{4}$. Then we have

$$
\begin{aligned}
& \mathrm{pf}_{12}=e_{34}, \quad \mathrm{pf}_{13}=-e_{24}, \quad \mathrm{pf}_{14}=e_{23}, \\
& \mathrm{pf}_{23}=e_{14}, \quad \mathrm{pf}_{24}=-e_{13}, \quad \mathrm{pf}_{34}=e_{12} .
\end{aligned}
$$

In other words, $\mathrm{pf}_{i j}$ is equal to $* e_{i j}$ for $i \neq j$, where we denote by $*$ the Hodge operator.

From the definition of $\mathrm{pf}_{i j}$, we have

Proposition 4.8. The elements $\left\{\mathrm{pf}_{i j}\right\}_{i, j}$ satisfy

$$
\mathrm{pf}_{i j}+\mathrm{pf}_{j i}=2 \delta_{i j} \mathrm{pf}
$$

and $\sum_{i} \mathrm{pf}_{i i}=2 \mathrm{mpf}$.

The equation (4.6) gives the anti-symmetric identity $\mathrm{pf}_{i j}-\delta_{i j} \mathrm{pf}=-\left(\mathrm{pf}_{j i}-\delta_{j i} \mathrm{pf}\right)$, the same as (3.14). This induces Bochner-Weitzenböck formulas in Section 6. Hence we call (4.6) the universal Bochner-Weitzenböck formula as well as (3.11).

Proposition 4.9. We can connect $\mathrm{pf}_{i j}$ to the Clifford homomorphisms $\left\{p_{\lambda}^{\rho}\right\}_{\lambda}$,

$$
\sum_{\lambda} \pi_{\lambda}(\mathrm{pf}) p_{\lambda}^{\rho}\left(e_{i}\right)^{*} p_{\lambda}^{\rho}\left(e_{j}\right)=\pi_{\rho}\left(\mathrm{pf}_{i j}\right)
$$

In particular, we have

$$
\sum_{\lambda} \pi_{\lambda}(\mathrm{pf}) \sum_{i} p_{\lambda}^{\rho}\left(e_{i}\right)^{*} p_{\lambda}^{\rho}\left(e_{i}\right)=2 m \pi_{\rho}(\mathrm{pf}) .
$$


Proof. For $\phi$ and $\psi$ in $V_{\rho}$,

$$
\begin{aligned}
& \left\langle\pi_{\rho} \otimes \pi_{\mu_{1}}(\mathrm{pf})\left(\phi \otimes e_{j}\right), \psi \otimes e_{i}\right\rangle=\sum_{\lambda}\left\langle\pi_{\lambda}(\mathrm{pf}) p_{\lambda}^{\rho}\left(e_{j}\right) \phi, p_{\lambda}^{\rho}\left(e_{i}\right) \psi\right\rangle \\
= & \left\langle\sum_{\lambda} \pi_{\lambda}(\mathrm{pf}) p_{\lambda}^{\rho}\left(e_{i}\right)^{*} p_{\lambda}^{\rho}\left(e_{j}\right) \phi, \psi\right\rangle .
\end{aligned}
$$

On the other hand, we know

$$
\begin{aligned}
& \pi_{\rho} \otimes \pi_{\mu_{1}}\left(e_{\sigma(2 l-1) \sigma(2 l)}\right)\left(\phi \otimes e_{j}\right) \\
= & \begin{cases}\pi_{\rho}\left(e_{\sigma(2 l-1) \sigma(2 l)}\right) \phi \otimes e_{j}+\phi \otimes e_{\sigma(2 l)}, & \sigma(2 l-1)=j, \\
\pi_{\rho}\left(e_{\sigma(2 l-1) \sigma(2 l)}\right) \phi \otimes e_{j}-\phi \otimes e_{\sigma(2 l-1)}, & \sigma(2 l)=j, \\
\pi_{\rho}\left(e_{\sigma(2 l-1) \sigma(2 l)}\right) \phi \otimes e_{j}, & \text { otherwise. }\end{cases}
\end{aligned}
$$

Then taking account of $\left\langle e_{k}, e_{l}\right\rangle=\delta_{k l}$, we have

$$
\begin{aligned}
& (\sqrt{-1})^{m} 2^{m} m !\left\langle\pi_{\rho} \otimes \pi_{\mu_{1}}(\mathrm{pf})\left(\phi \otimes e_{j}\right), \psi \otimes e_{i}\right\rangle \\
= & \left\langle\pi_{\rho} \otimes \pi_{\mu_{1}}\left(\sum_{\sigma \in \mathfrak{S}_{2 m}} \operatorname{sgn}(\sigma) e_{\sigma(1) \sigma(2)} \cdots e_{\sigma(2 m-1) \sigma(2 m)}\right)\left(\phi \otimes e_{j}\right), \psi \otimes e_{i}\right\rangle \\
= & \begin{cases}(\sqrt{-1})^{m} 2^{m} m !\left\langle\pi_{\rho}(\mathrm{pf}) \phi, \psi\right\rangle, & i=j, \\
(-1)^{i+j} 2 m\left\langle\pi_{\rho}\left(\sum_{\sigma \in \mathfrak{S}_{2 m}^{i j}} \operatorname{sgn}(\sigma) e_{\sigma(1) \sigma(2)} \cdots e_{\sigma(2 m-1) \sigma(2 m)}\right) \phi, \psi\right\rangle, & i<j, \\
(-1)^{i+j+1} 2 m\left\langle\pi_{\rho}\left(\sum_{\sigma \in \mathfrak{S}_{2 m}^{i j}} \operatorname{sgn}(\sigma) e_{\sigma(1) \sigma(2)} \cdots e_{\sigma(2 m-1) \sigma(2 m)}\right) \phi, \psi\right\rangle, & i>j .\end{cases}
\end{aligned}
$$

Combining this equation and (4.9), we have (4.7). The equation (4.8) is clear from (4.6).

We consider the exceptional case. It follows from (3.6) that $\pi_{\lambda_{+}}(\mathrm{pf})=-\pi_{\lambda_{-}}$(pf) $\neq 0$ and $\pi_{\lambda}$ (pf) $=0$ for $\lambda \neq \lambda_{ \pm}$. Then

$$
p_{\lambda_{+}}^{\rho}\left(e_{i}\right)^{*} p_{\lambda_{+}}^{\rho}\left(e_{j}\right)-p_{\lambda_{-}}^{\rho}\left(e_{i}\right)^{*} p_{\lambda_{-}}^{\rho}\left(e_{j}\right)=\frac{1}{\pi_{\lambda_{+}}(\mathrm{pf})} \pi_{\rho}\left(\mathrm{pf}_{i j}\right) .
$$

As a result, we have the following corollary of Propositions 4.4 and 4.9 ,

Corollary 4.10. We can realize $p_{\lambda}^{\rho}\left(e_{i}\right)^{*} p_{\lambda}^{\rho}\left(e_{j}\right)$ as a linear combination of $\left\{\pi_{\rho}\left(e_{i j}^{q}\right)\right\}_{q}$ and $\pi_{\rho}\left(\mathrm{pf}_{i j}\right)$.

This corollary implies that we may investigate $e_{i j}^{q}$ and $\mathrm{pf}_{i j}$ instead of Clifford homomorphisms. In fact, the universal Bochner-Weitzenböck formulas (3.11) and (4.6) give a lot of identities for Clifford homomorphisms in the following way.

We consider the equation (4.2) for $q=0,1$. Because of $e_{i j}=-e_{j i}$, we have

$$
\begin{gathered}
\sum_{\lambda}\left(p_{\lambda}^{\rho}\left(e_{i}\right)^{*} p_{\lambda}^{\rho}\left(e_{j}\right)+p_{\lambda}^{\rho}\left(e_{j}\right)^{*} p_{\lambda}^{\rho}\left(e_{i}\right)\right)=2 \delta_{i j}, \\
\sum_{\lambda} w(\rho ; \lambda)\left(p_{\lambda}^{\rho}\left(e_{i}\right)^{*} p_{\lambda}^{\rho}\left(e_{j}\right)+p_{\lambda}^{\rho}\left(e_{j}\right)^{*} p_{\lambda}^{\rho}\left(e_{i}\right)\right)=0 .
\end{gathered}
$$

Note that the Clifford relation on spinor space follows from the above two equations. To construct further identities, we use (3.11) and (4.6). We define the translated 
conformal weight $\hat{w}(\rho ; \lambda)$ by

$$
\hat{w}(\rho ; \lambda):=w(\rho ; \lambda)+\frac{n-1}{2} .
$$

Then it is simple to show that

$$
\sum_{\lambda} \hat{w}(\rho ; \lambda)^{q} p_{\lambda}^{\rho}\left(e_{i}\right)^{*} p_{\lambda}^{\rho}\left(e_{j}\right)=\pi_{\rho}\left(\hat{e}_{i j}^{q}\right) .
$$

Substituting (3.12) for this equation, we have

$$
\begin{aligned}
& \sum_{\lambda} \hat{w}(\rho ; \lambda)^{2 q} p_{\lambda}^{\rho}\left(e_{i}\right)^{*} p_{\lambda}^{\rho}\left(e_{j}\right) \\
= & \sum_{\lambda}\left\{\hat{w}(\rho ; \lambda)^{2 q}+\sum_{p=0}^{2 q-1}(-\hat{w}(\rho ; \lambda))^{p} \pi_{\rho}\left(\hat{c}_{2 q-1-p}\right)\right\} p_{\lambda}^{\rho}\left(e_{j}\right)^{*} p_{\lambda}^{\rho}\left(e_{i}\right),
\end{aligned}
$$

and hence,

$$
\sum_{\lambda}\left\{\sum_{p=0}^{2 q-1}(-\hat{w}(\rho ; \lambda))^{p} \pi_{\rho}\left(\hat{c}_{2 q-1-p}\right)\right\}\left(p_{\lambda}^{\rho}\left(e_{i}\right)^{*} p_{\lambda}^{\rho}\left(e_{j}\right)+p_{\lambda}^{\rho}\left(e_{j}\right)^{*} p_{\lambda}^{\rho}\left(e_{i}\right)\right)=0 .
$$

From (4.6) and (4.7) for $n=2 m$, we also have

$$
\sum_{\lambda} \pi_{\lambda}(\mathrm{pf})\left(p_{\lambda}^{\rho}\left(e_{i}\right)^{*} p_{\lambda}^{\rho}\left(e_{j}\right)+p_{\lambda}^{\rho}\left(e_{j}\right)^{*} p_{\lambda}^{\rho}\left(e_{i}\right)\right)=2 \pi_{\rho}(\mathrm{pf}) \delta_{i j} .
$$

We can easily show from (3.6) and (4.4) that

$$
(w(\rho ; \lambda)+m-1) \pi_{\lambda}(\mathrm{pf})=(w(\rho ; \lambda)+m) \pi_{\rho}(\mathrm{pf})
$$

for each $\lambda$. Then

$$
\begin{aligned}
& \sum_{\lambda} \pi_{\lambda}(\mathrm{pf}) w(\rho ; \lambda)\left(p_{\lambda}^{\rho}\left(e_{i}\right)^{*} p_{\lambda}^{\rho}\left(e_{j}\right)+p_{\lambda}^{\rho}\left(e_{j}\right)^{*} p_{\lambda}^{\rho}\left(e_{i}\right)\right) \\
= & \sum_{\lambda}\left(-(m-1) \pi_{\lambda}(\mathrm{pf})+w(\rho ; \lambda) \pi_{\rho}(\mathrm{pf})+m \pi_{\rho}(\mathrm{pf})\right)\left(p_{\lambda}^{\rho}\left(e_{i}\right)^{*} p_{\lambda}^{\rho}\left(e_{j}\right)+p_{\lambda}^{\rho}\left(e_{j}\right)^{*} p_{\lambda}^{\rho}\left(e_{i}\right)\right) \\
= & -2(m-1) \pi_{\rho}(\mathrm{pf}) \delta_{i j}+2 m \pi_{\rho}(\mathrm{pf}) \delta_{i j}=2 \pi_{\rho}(\mathrm{pf}) \delta_{i j} .
\end{aligned}
$$

As a result, we have

$$
\sum_{\lambda} \pi_{\lambda}(\mathrm{pf})(w(\rho ; \lambda)-1)\left(p_{\lambda}^{\rho}\left(e_{i}\right)^{*} p_{\lambda}^{\rho}\left(e_{j}\right)+p_{\lambda}^{\rho}\left(e_{j}\right)^{*} p_{\lambda}^{\rho}\left(e_{i}\right)\right)=0
$$

Thus we obtain algebraic relations among Clifford homomorphisms like the Clifford relation.

Theorem 4.11. Let $\xi$ and $\eta$ be in $\mathbb{R}^{n}$. Then the Clifford homomorphisms $\left\{p_{\lambda}^{\rho}\right\}_{\lambda}$ satisfy

$$
\begin{gathered}
\sum_{\lambda}\left(p_{\lambda}^{\rho}(\xi)^{*} p_{\lambda}^{\rho}(\eta)+p_{\lambda}^{\rho}(\eta)^{*} p_{\lambda}^{\rho}(\xi)\right)=2\langle\xi, \eta\rangle \\
\sum_{\lambda}\left\{\sum_{p=0}^{2 q-1}(-\hat{w}(\rho ; \lambda))^{p} \pi_{\rho}\left(\hat{c}_{2 q-1-p}\right)\right\}\left(p_{\lambda}^{\rho}(\xi)^{*} p_{\lambda}^{\rho}(\eta)+p_{\lambda}^{\rho}(\eta)^{*} p_{\lambda}^{\rho}(\xi)\right)=0
\end{gathered}
$$


and, for $n=2 m$,

$$
\begin{aligned}
& \sum_{\lambda} \pi_{\lambda}(\mathrm{pf})\left(p_{\lambda}^{\rho}(\xi)^{*} p_{\lambda}^{\rho}(\eta)+p_{\lambda}^{\rho}(\eta)^{*} p_{\lambda}^{\rho}(\xi)\right)=2 \pi_{\rho}(\mathrm{pf})\langle\xi, \eta\rangle, \\
& \sum_{\lambda} \pi_{\lambda}(\mathrm{pf})(w(\rho ; \lambda)-1)\left(p_{\lambda}^{\rho}(\xi)^{*} p_{\lambda}^{\rho}(\eta)+p_{\lambda}^{\rho}(\eta)^{*} p_{\lambda}^{\rho}(\xi)\right)=0 .
\end{aligned}
$$

In the rest of this section, we calculate the eigenvalues of $c_{q}$ on irreducible $\mathfrak{s o}(n)$ modules. Our method is based on [9] and 21]. It is known that the usual Clifford multiplication satisfies $\left(\pi_{\mu_{1}}(g) \xi\right) \cdot=\pi_{\Delta}(g) \xi \cdot \pi_{\Delta}\left(g^{-1}\right)$ for $g$ in $\operatorname{Spin}(n)$ and $\xi$ in $\mathbb{R}^{n}$. The Clifford homomorphism is also compatible with the action of $\mathrm{SO}(n)$ or $\operatorname{Spin}(n)$.

Lemma 4.12. For $g$ in $\mathrm{SO}(n)$ or $\operatorname{Spin}(n)$ and $\xi$ in $\mathbb{C}^{n}$, we have

$$
p_{\lambda}^{\rho}\left(\pi_{\mu_{1}}(g) \xi\right)=\pi_{\lambda}(g) p_{\lambda}^{\rho}(\xi) \pi_{\rho}\left(g^{-1}\right) .
$$

Hence, for $e_{i j}$ in $\mathfrak{s o}(n)$,

$$
p_{\lambda}^{\rho}\left(\pi_{\mu_{1}}\left(e_{i j}\right) \xi\right)=\pi_{\lambda}\left(e_{i j}\right) p_{\lambda}^{\rho}(\xi)-p_{\lambda}^{\rho}(\xi) \pi_{\rho}\left(e_{i j}\right) .
$$

Proof. Consider the action of $g$ on $V_{\rho} \otimes \mathbb{C}^{n}=\bigoplus_{\lambda} V_{\lambda}$, and we have

$$
\sum_{\lambda} \pi_{\lambda}(g) p_{\lambda}^{\rho}(\xi) \phi=\pi_{\rho} \otimes \pi_{\mu_{1}}(g)(\phi \otimes \xi)=\pi_{\rho}(g) \otimes \pi_{\mu_{1}}(g) \xi=\sum_{\lambda} p_{\lambda}^{\rho}\left(\pi_{\mu_{1}}(g) \xi\right) \pi_{\rho}(g) \phi
$$

for $\phi \otimes \xi$ in $V_{\rho} \otimes \mathbb{C}^{n}$. Then we conclude that $p_{\lambda}^{\rho}\left(\pi_{\mu_{1}}(g) \xi\right)=\pi_{\lambda}(g) p_{\lambda}^{\rho}(\xi) \pi_{\rho}\left(g^{-1}\right)$.

The Clifford homomorphism $p_{\lambda}^{\rho}$ is defined through the projection $\Pi_{\lambda}^{\rho}: V_{\rho} \otimes \mathbb{C}^{n} \rightarrow$ $V_{\lambda}$. Therefore $\Pi_{\lambda}^{\rho}$ is realized with the Clifford homomorphism.

Lemma 4.13. The orthogonal projection $\Pi_{\lambda}^{\rho}: V_{\rho} \otimes \mathbb{C}^{n} \rightarrow V_{\lambda} \subset V_{\rho} \otimes \mathbb{C}^{n}$ is realized as follows:

$$
\Pi_{\lambda}^{\rho}(\phi \otimes \xi)=\sum_{i} p_{\lambda}^{\rho}\left(e_{i}\right)^{*} p_{\lambda}^{\rho}(\xi) \phi \otimes e_{i} .
$$

Proof. It follows from (4.16) that the following mapping is an $\mathfrak{s o}(n)$-equivariant injection,

$$
V_{\lambda} \ni \psi \mapsto \sum_{i} p_{\lambda}^{\rho}\left(e_{i}\right)^{*} \psi \otimes e_{i} \in V_{\rho} \otimes \mathbb{C}^{n} .
$$

Taking into account (4.3), we decompose $\phi \otimes \xi$ as

$$
\phi \otimes \xi=\sum_{i}\left\langle\xi, e_{i}\right\rangle \phi \otimes e_{i}=\sum_{i} \sum_{\lambda} p_{\lambda}^{\rho}\left(e_{i}\right)^{*} p_{\lambda}^{\rho}(\xi) \phi \otimes e_{i}=\sum_{\lambda} \sum_{i} p_{\lambda}^{\rho}\left(e_{i}\right)^{*} p_{\lambda}^{\rho}(\xi) \phi \otimes e_{i} .
$$

Since $\sum_{i} p_{\lambda}^{\rho}\left(e_{i}\right)^{*} p_{\lambda}^{\rho}(\xi) \phi \otimes e_{i}$ is in $V_{\lambda}$ for each $\lambda$, we have the projection formula (4.18).

Lemma 4.12 implies that $\sum_{i} p_{\lambda}^{\rho}\left(e_{i}\right)^{*} p_{\lambda}^{\rho}\left(e_{i}\right)$ is invariant under the action of $\mathfrak{s o}(n)$ and constant on $V_{\rho}$.

Proposition 4.14. We set $d(\rho):=\operatorname{dim} V_{\rho}$. Then

$$
\sum_{i} p_{\lambda}^{\rho}\left(e_{i}\right)^{*} p_{\lambda}^{\rho}\left(e_{i}\right)=d(\lambda) / d(\rho)
$$


The eigenvalues of $c_{q}$ and $\hat{c}_{q}$ on irreducible $\mathfrak{s o}(n)$-module $V_{\rho}$ are

$$
\pi_{\rho}\left(c_{q}\right)=\frac{1}{d(\rho)} \sum_{\lambda} w(\rho ; \lambda)^{q} d(\lambda), \quad \pi_{\rho}\left(\hat{c}_{q}\right)=\frac{1}{d(\rho)} \sum_{\lambda} \hat{w}(\rho ; \lambda)^{q} d(\lambda) .
$$

Moreover, we have a relation for eigenvalues of the Pfaffian element,

$$
2 m \pi_{\rho}(\mathrm{pf})=\frac{1}{d(\rho)} \sum_{\lambda} \pi_{\lambda}(\mathrm{pf}) d(\lambda)=\frac{1}{d(\rho)} \sum_{\lambda} \pi_{\lambda}(\mathrm{pf}) w(\rho ; \lambda) d(\lambda) .
$$

Proof. Let $\left\{\phi_{\alpha}\right\}_{\alpha=1}^{\operatorname{dim} V_{\rho}}$ be an orthonormal basis of $V_{\rho}$. Taking the trace of $\Pi_{\lambda}^{\rho}$, we have

$$
\begin{aligned}
d(\lambda) & =\sum_{\alpha, i}\left\langle\Pi_{\lambda}^{\rho}\left(\phi_{\alpha} \otimes e_{i}\right), \phi_{\alpha} \otimes e_{i}\right\rangle=\sum_{\alpha, i, j}\left\langle p_{\lambda}^{\rho}\left(e_{j}\right)^{*} p_{\lambda}^{\rho}\left(e_{i}\right)\left(\phi_{\alpha}\right) \otimes e_{j}, \phi_{\alpha} \otimes e_{i}\right\rangle \\
& =\sum_{\alpha, i, j}\left\langle p_{\lambda}^{\rho}\left(e_{j}\right)^{*} p_{\lambda}^{\rho}\left(e_{i}\right)\left(\phi_{\alpha}\right), \phi_{\alpha}\right\rangle \delta_{i j}=\sum_{\alpha}\left\langle\phi_{\alpha}, \phi_{\alpha}\right\rangle \sum_{i} p_{\lambda}^{\rho}\left(e_{i}\right)^{*} p_{\lambda}^{\rho}\left(e_{i}\right) \\
& =d(\rho) \sum_{i} p_{\lambda}^{\rho}\left(e_{i}\right)^{*} p_{\lambda}^{\rho}\left(e_{i}\right) .
\end{aligned}
$$

Thus we obtain $\sum_{i} p_{\lambda}^{\rho}\left(e_{i}\right)^{*} p_{\lambda}^{\rho}\left(e_{i}\right)=d(\lambda) / d(\rho)$ and easily show (4.19) and (4.20).

Calculating $d(\rho)$ by Weyl's dimension formula, we have an explicit formula for $\pi_{\rho}\left(c_{q}\right)$ in [20] and 21]. Though it is not easy to compute $d(\lambda)$ and $d(\rho)$ separately, D. Calderbank, P. Gauduchon and M. Herzlich show that the relative dimension $d(\lambda) / d(\rho)$ can be calculated more easily [9]. We denote by $\left\{\lambda_{i}\right\}_{i=1}^{N}$ the highest weights in $V_{\rho} \otimes \mathbb{C}^{n}$. From Proposition 4.1, we show that $N$ is even only in the following case: (1) $n$ is even and $\rho^{m} \neq 0$, (2) $n$ is odd and $\rho^{m}=1 / 2$, (3) the exceptional case. By a straightforward calculation, we have

Proposition 4.15 ([9, Lemma 4.5]).

(1) If $N$ is odd,

$$
\frac{d\left(\lambda_{j}\right)}{d(\rho)}=\left(2 \hat{w}\left(\rho ; \lambda_{j}\right)+1\right) \prod_{k \neq j} \frac{\hat{w}\left(\rho ; \lambda_{j}\right)+\hat{w}\left(\rho ; \lambda_{k}\right)}{\hat{w}\left(\rho ; \lambda_{j}\right)-\hat{w}\left(\rho ; \lambda_{k}\right)} .
$$

(2) If $N$ is even,

$$
\frac{d\left(\lambda_{j}\right)}{d(\rho)}=\left(2 \hat{w}\left(\rho ; \lambda_{j}\right)-1\right) \prod_{k \neq j} \frac{\hat{w}\left(\rho ; \lambda_{j}\right)+\hat{w}\left(\rho ; \lambda_{k}\right)}{\hat{w}\left(\rho ; \lambda_{j}\right)-\hat{w}\left(\rho ; \lambda_{k}\right)} .
$$

This proposition gives an interesting relation between the conformal weights and the relative dimensions, which is used in Section 7 .

\section{Corollary 4.16.}

$$
\sum_{j} \frac{1}{2 \hat{w}\left(\rho ; \lambda_{j}\right)-(-1)^{N}} \frac{d\left(\lambda_{j}\right)}{d(\rho)}= \begin{cases}1 & \text { if } N \text { is odd } \\ 0 & \text { if } N \text { is even } .\end{cases}
$$




\section{Gradients on Riemannian Manifolds}

In this section, we define gradients and study their fundamental properties. We consider only gradients on Riemannian manifolds. The spin case is left to the readers, where the spin connection is used instead of the Levi-Civita connection [11, 19].

Let $(M, g)$ be an $n$-dimensional oriented Riemannian manifold and let $\mathbf{S O}(M)$ be the principal $\mathrm{SO}(n)$ bundle of the oriented orthonormal frames on $M$. For an irreducible unitary representation $\left(\pi_{\rho}, V_{\rho}\right)$ of $\mathrm{SO}(n)$, we have an associated Hermitian vector bundle $\mathbf{S}_{\rho}:=\mathbf{S O}(M) \times_{\pi_{\rho}} V_{\rho}$. The Levi-Civita connection on $\mathbf{S O}(M)$ gives a covariant derivative $\nabla$ on $\mathbf{S}_{\rho}$ compatible with fiber metric as follows. Let $e=\left(e_{1}, \cdots, e_{n}\right)$ be a local section of $\mathbf{S O}(M)$. For a unitary basis $\left\{\phi_{\alpha}\right\}_{\alpha}$ of $V_{\rho}$, we have a local frame $\left\{\left[e, \phi_{\alpha}\right]\right\}_{\alpha}$ of $\mathbf{S}_{\rho}$. With respect to this local trivialization, the covariant derivative $\nabla$ is defined to be

$$
\nabla:=d+\frac{1}{2} \sum_{i j} g\left(\nabla^{T} e_{i}, e_{j}\right) \pi_{\rho}\left(e_{i j}\right)
$$

where $\nabla^{T}$ is the Levi-Civita connection on the tangent bundle $T(M)$. Since the connection 1-form is skew Hermitian, the derivative $\nabla$ is compatible with fiber metric, that is, $X\langle\phi, \psi\rangle=\left\langle\nabla_{X} \phi, \psi\right\rangle+\left\langle\phi, \nabla_{X} \psi\right\rangle$ for every vector field $X$.

We shall extend Clifford homomorphisms to bundle homomorphisms. We consider the tensor bundle $\mathbf{S}_{\rho} \otimes T_{\mathbb{C}}(M)=\mathbf{S}_{\rho} \otimes(T(M) \otimes \mathbb{C})$ and decompose it as

$$
\mathbf{S}_{\rho} \otimes T_{\mathbb{C}}(M)=\bigoplus_{\lambda} \mathbf{S}_{\lambda}
$$

For each vector field $X=\sum X^{i} e_{i}$, we define a bundle homomorphism $p_{\lambda}^{\rho}(X)$ in $\Gamma\left(M, \operatorname{Hom}\left(\mathbf{S}_{\rho}, \mathbf{S}_{\lambda}\right)\right)$ by

$$
p_{\lambda}^{\rho}(X): \mathbf{S}_{\rho} \ni[e, \phi] \mapsto \sum_{i} X^{i}\left[e, p_{\lambda}^{\rho}\left(e_{i}\right) \phi\right] \in \mathbf{S}_{\lambda} .
$$

From (4.16), we know that this bundle homomorphism is well defined. Furthermore, we can show from (4.17) and (5.1) that

$$
\nabla_{Y}\left(p_{\lambda}^{\rho}(X) \phi\right)=p_{\lambda}^{\rho}\left(\nabla_{X}^{T} Y\right) \phi+p_{\lambda}^{\rho}(X) \nabla_{Y} \phi
$$

for $\phi$ in $\Gamma\left(M, \mathbf{S}_{\rho}\right)$.

We define geometric first order differential operators depending on the Riemannian metric $g$ on each associated bundle.

Definition 5.1. Decomposing $\nabla$ along (5.2), we have the first order differential operator $D_{\lambda}^{\rho}=\Pi_{\lambda}^{\rho} \circ \nabla$

$$
D_{\lambda}^{\rho}: \Gamma\left(M, \mathbf{S}_{\rho}\right) \stackrel{\nabla}{\longrightarrow} \Gamma\left(M, \mathbf{S}_{\rho} \otimes T_{\mathbb{C}}^{*}(M)\right) \stackrel{\simeq}{\longrightarrow} \Gamma\left(M, \mathbf{S}_{\rho} \otimes T_{\mathbb{C}}(M)\right) \stackrel{\Pi_{\lambda}^{\rho}}{\longrightarrow} \Gamma\left(M, \mathbf{S}_{\lambda}\right),
$$

for each $\lambda$. Here $\Pi_{\lambda}^{\rho}$ is the orthogonal projection defined fiberwise from $\mathbf{S}_{\rho} \otimes T_{\mathbb{C}}(M)$ onto $\mathbf{S}_{\lambda}$. We call this first order differential operator $D_{\lambda}^{\rho}$ the gradient associated to $\rho$ and $\lambda$.

Example 5.2. Let $\mathbf{S}_{\Delta}$ be the spinor bundle, where $n=2 m+1$ and $\Delta=\left((1 / 2)_{m}\right)$. We have the irreducible decomposition $\mathbf{S}_{\Delta} \otimes T_{\mathbb{C}}(M)=\mathbf{S}_{T} \oplus \mathbf{S}_{\Delta}$, where $T=$ $\left(3 / 2,(1 / 2)_{m-1}\right)$. Then $D_{\Delta}^{\Delta}$ is the Dirac operator and $D_{T}^{\Delta}$ is the twistor operator up to a normalization. 
Example 5.3. The bundle $\Lambda^{p}(M)$ of (complex) differential forms is realized as an associated vector bundle,

$$
\begin{cases}\Lambda_{ \pm}^{m}(M)=\mathbf{S}_{\left(1_{m-1}, \pm 1\right)} & \text { for } n=2 m \text { and } p=m, \\ \Lambda^{p}(M) \simeq \Lambda^{n-p}(M)=\mathbf{S}_{\left(1_{p}\right)} & \text { otherwise. }\end{cases}
$$

On this vector bundle, we have three gradients, the conformal Killing operator $C$, the exterior derivative $d$, and the interior derivative $d^{*}$ up to a normalization.

Because the principal symbol of $D_{\lambda}^{\rho}$ is the Clifford homomorphism $p_{\lambda}^{\rho}$, we have a formula of the gradient $D_{\lambda}^{\rho}$,

$$
D_{\lambda}^{\rho}(\phi)=\Pi_{\lambda}^{\rho}\left(\sum_{i} \nabla_{e_{i}} \phi \otimes e_{i}^{*}\right)=\sum_{i} p_{\lambda}^{\rho}\left(e_{i}\right) \nabla_{e_{i}} \phi
$$

From a similar discussion to the Dirac operator [19], we show that the formal adjoint operator $\left(D_{\lambda}^{\rho}\right)^{*}$ of $D_{\lambda}^{\rho}$ is

$$
\left(D_{\lambda}^{\rho}\right)^{*}=-\sum_{i} p_{\lambda}^{\rho}\left(e_{i}\right)^{*} \nabla_{e_{i}} .
$$

An important feature of the Dirac operator is conformal covariance. The gradients are also conformally covariant operators. Though this fact has been shown by H. D. Fegan [10], we give an explicit proof. We deform the Riemannian metric $g$ conformally as $g^{\prime}=\exp (2 \sigma) g$ for $\sigma$ in $C^{\infty}(M)$. We denote the objects associated to $g^{\prime}$ by adding the symbol " $"$ " to them. The orthonormal frame bundle $\mathbf{S O}(M)$ is isomorphic to $\mathbf{S O}^{\prime}(M)$,

$$
\Phi: \mathbf{S O}(M) \ni e=\left(e_{1}, \cdots, e_{n}\right) \mapsto e^{\prime}=e^{-\sigma}\left(e_{1}, \cdots, e_{n}\right) \in \mathbf{S O}^{\prime}(M) .
$$

Then there is a bundle isometry for each $\rho$,

$$
\Phi_{\rho}: \mathbf{S}_{\rho}=\mathbf{S O}(M) \times_{\pi_{\rho}} V_{\rho} \ni[e, \phi] \mapsto\left[e^{\prime}, \phi\right] \in \mathbf{S O}^{\prime}(M) \times_{\pi_{\rho}} V_{\rho}=\mathbf{S}_{\rho}^{\prime}
$$

such that $\Phi_{\lambda} \circ p_{\lambda}^{\rho}(X)=e^{-\sigma} p_{\lambda}^{\rho}(X) \circ \Phi_{\rho}$ for each vector $X$. The Levi-Civita connection $\nabla^{T}$ on $T(M)$ changes as

$$
\nabla^{T}{ }_{X}^{\prime} Y=\nabla_{X}^{T} Y+(X \sigma) Y+(Y \sigma) X-g(X, Y) \operatorname{grad}(\sigma),
$$

where $\operatorname{grad}(\sigma):=\sum\left(e_{i} \sigma\right) e_{i}$ is the gradient vector field of $\sigma$ with respect to $g$. We show from (5.1) that the covariant derivative on $\mathbf{S}_{\rho}$ changes as

$$
\nabla_{X}^{\prime} \Phi_{\rho}(\phi)-\Phi_{\rho}\left(\nabla_{X} \phi\right)=\Phi_{\rho}\left(\frac{1}{2} \sum_{i j}\left(\left(e_{i} \sigma\right) g\left(X, e_{j}\right)-\left(e_{j} \sigma\right) g\left(X, e_{i}\right)\right) \pi_{\rho}\left(e_{i j}\right) \phi\right)
$$

for $\phi$ in $\Gamma\left(M, \mathbf{S}_{\rho}\right)$. It follows from (4.1) and (5.4) that

$$
\begin{aligned}
& D_{\lambda}^{\prime \rho} \Phi_{\rho}(\phi) \\
= & \Pi_{\lambda}^{\rho}\left(\sum_{i} \nabla_{e_{i}^{\prime}}^{\prime} \Phi_{\rho}(\phi) \otimes\left(e_{i}^{\prime}\right)^{*}\right)=\sum_{i} \Pi_{\lambda}^{\rho}\left(\nabla_{e_{i}}^{\prime} \Phi_{\rho}(\phi) \otimes\left(e_{i}\right)^{*}\right)=\sum_{i} p_{\lambda}^{\rho}\left(e_{i}\right) \nabla_{e_{i}}^{\prime} \Phi_{\rho}(\phi) \\
= & e^{-\sigma} \Phi_{\lambda}\left(D_{\lambda}^{\rho} \phi+\frac{1}{2} \sum_{i k l} p_{\lambda}^{\rho}\left(e_{i}\right)\left(\left(e_{k} \sigma\right) g\left(e_{i}, e_{l}\right)-\left(e_{l} \sigma\right) g\left(e_{i}, e_{k}\right)\right) \pi_{\rho}\left(e_{k l}\right) \phi\right) \\
= & e^{-\sigma} \Phi_{\lambda}\left(D_{\lambda}^{\rho} \phi-w(\rho ; \lambda) p_{\lambda}^{\rho}(\operatorname{grad}(\sigma)) \phi\right) .
\end{aligned}
$$


Here, precisely speaking, $D_{\lambda}^{\rho}$ is defined through not $\Pi_{\lambda}^{\rho}: \mathbf{S}_{\rho} \otimes T_{\mathbb{C}}(M) \rightarrow \mathbf{S}_{\lambda}$ but $\Pi_{\lambda}^{\rho}: \mathbf{S}_{\rho} \otimes T_{\mathbb{C}}^{*}(M) \rightarrow \mathbf{S}_{\lambda}$. Therefore we use $\Phi_{\lambda} \circ p_{\lambda}^{\rho}\left(e_{i}\right)=e^{\sigma} p_{\lambda}^{\rho}\left(e_{i}\right) \circ \Phi_{\rho}$ in the above equation. We also have

$$
\left[D_{\lambda}^{\rho}, f\right]=D_{\lambda}^{\rho} \circ f-f D_{\lambda}^{\rho}=p_{\lambda}^{\rho}(\operatorname{grad}(f))
$$

for $f$ in $C^{\infty}(M)$. The equations (5.5) and (5.6) give conformal covariance of $D_{\lambda}^{\rho}$. The next proposition answers why we call $w(\rho ; \lambda)$ the conformal weight.

Proposition 5.4 ([10, Theorem 1.1]). When we change the Riemannian metric $g$ to $g^{\prime}=e^{2 \sigma} g$, the gradient $D_{\lambda}^{\rho}$ changes as

$$
D_{\lambda}^{\prime \rho}=\left(e^{(w(\rho ; \lambda)-1) \sigma} \Phi_{\lambda}\right) \circ D_{\lambda}^{\rho} \circ\left(e^{w(\rho ; \lambda) \sigma} \Phi_{\rho}\right)^{-1} .
$$

In particular, if the dimension of $\operatorname{ker} D_{\lambda}^{\rho}$ is finite, then $\operatorname{dim} \operatorname{ker} D_{\lambda}^{\rho}$ is a conformal invariant of $M$.

\section{Curvature endomorphisms}

Let $R_{T}$ be the Riemannian curvature on $T(M)$. For a local oriented orthonormal frame $e=\left(e_{1}, \cdots, e_{n}\right)$, we set a local expression of $R_{T}$ by $R_{i j k l}:=g\left(R_{T}\left(e_{i}, e_{j}\right) e_{k}, e_{l}\right)$, and denote the Ricci tensor by $R_{i j}=\sum_{k} R_{i k k j}$ and the scalar curvature by $\kappa=$ $\sum_{i} R_{i i}$. We decompose the Riemannian curvature $R_{i j k l}$,

$$
R_{i j k l}=W_{i j k l}+K_{i j k l}+S_{i j k l},
$$

where

$$
\begin{aligned}
S_{i j k l} & :=\frac{\kappa}{n(n-1)}\left(\delta_{i l} \delta_{j k}-\delta_{i k} \delta_{j l}\right), \\
E_{i j} & :=\frac{1}{n-2}\left(\frac{\kappa}{n} \delta_{i j}-R_{i j}\right), \\
K_{i j k l} & :=E_{i k} \delta_{j l}+E_{j l} \delta_{i k}-E_{i l} \delta_{j k}-E_{j k} \delta_{i l}, \\
W_{i j k l} & :=R_{i j k l}-E_{i j k l}-S_{i j k l} .
\end{aligned}
$$

The conformal Weyl tensor $W_{i j k l}$ and the Einstein tensor $E_{i j}$ satisfy

$$
\sum_{i} W_{i j i l}=0, \quad E_{i j}=E_{j i}, \quad \sum_{i} E_{i i}=0 .
$$

We shall discuss curvature endomorphisms on the associate vector bundle $\mathbf{S}_{\rho}$. We define the second order derivative $\nabla_{X, Y}^{2}$ on $\mathbf{S}_{\rho}$ for vector fields $X$ and $Y$ by

$$
\nabla_{X, Y}^{2}:=\nabla_{X} \nabla_{Y}-\nabla_{\nabla_{X}^{T} Y}
$$

Then the curvature on $\mathbf{S}_{\rho}$ is $R_{\rho}(X, Y)=\nabla_{X, Y}^{2}-\nabla_{Y, X}^{2}$. From (5.1), a local expression of $R_{\rho}$ is

$$
R_{\rho}\left(e_{i}, e_{j}\right)=\frac{1}{2} \sum_{i j} R_{i j k l} \pi_{\rho}\left(e_{k l}\right) .
$$

By an easy calculation, we can decompose the curvature $R_{\rho}$,

$R_{\rho}\left(e_{i}, e_{j}\right)=\frac{1}{2} \sum_{k l} W_{i j k l} \pi_{\rho}\left(e_{k l}\right)+\sum_{k}\left(E_{i k} \pi_{\rho}\left(e_{k j}\right)-E_{j k} \pi_{\rho}\left(e_{k i}\right)\right)-\frac{\kappa}{n(n-1)} \pi_{\rho}\left(e_{i j}\right)$. 
Definition 6.1. We define a curvature endomorphism in $\Gamma\left(M, \operatorname{End}\left(\mathbf{S}_{\rho}\right)\right)$ by

$$
R_{\rho}^{q}:=\sum_{i j} \pi_{\rho}\left(e_{i j}^{q}\right) R_{\rho}\left(e_{i}, e_{j}\right)
$$

for each $q$. When $n$ is even,

$$
R_{\rho}^{\mathrm{pf}}:=\sum_{i j} \pi_{\rho}\left(\mathrm{pf}_{i j}\right) R_{\rho}\left(e_{i}, e_{j}\right) .
$$

Instead of $R_{\rho}^{q}$, we often use the translated curvature endomorphism

$$
\hat{R}_{\rho}^{q}:=\sum_{i j} \pi_{\rho}\left(\hat{e}_{i j}^{q}\right) R_{\rho}\left(e_{i}, e_{j}\right)=\sum_{0 \leq p \leq q}\left(\begin{array}{l}
q \\
p
\end{array}\right)\left(\frac{n-1}{2}\right)^{q-p} R_{\rho}^{p} .
$$

Example 6.2. $R_{\left((1 / 2)_{m-1}, \pm 1 / 2\right)}^{1}$ is $\kappa / 4$, and $R_{(1)}^{1} / 2$ is the Ricci transformation.

By Clifford homomorphisms, the curvature endomorphisms are rewritten as

$$
\begin{aligned}
& R_{\rho}^{q}=\sum_{i j, \lambda} w(\rho ; \lambda)^{q} p_{\lambda}^{\rho}\left(e_{i}\right)^{*} p_{\lambda}^{\rho}\left(e_{j}\right) R_{\rho}\left(e_{i}, e_{j}\right), \\
& R_{\rho}^{\mathrm{pf}}=\sum_{i j, \lambda} \pi_{\lambda}(\mathrm{pf}) p_{\lambda}^{\rho}\left(e_{i}\right)^{*} p_{\lambda}^{\rho}\left(e_{j}\right) R_{\rho}\left(e_{i}, e_{j}\right) .
\end{aligned}
$$

Proposition 6.3. The curvature endomorphisms $R_{\rho}^{q}$ and $R_{\rho}^{\mathrm{pf}}$ are self-adjoint endomorphisms of $\mathbf{S}_{\rho}$.

Proof. We consider a curvature endomorphism of $\mathbf{S}_{\rho}$,

$$
R_{\rho}(\lambda):=\sum_{i j} p_{\lambda}^{\rho}\left(e_{i}\right)^{*} p_{\lambda}^{\rho}\left(e_{j}\right) R_{\rho}\left(e_{i}, e_{j}\right)=\sum_{i j k l} R_{i j k l} p_{\lambda}^{\rho}\left(e_{i}\right)^{*} p_{\lambda}^{\rho}\left(e_{j}\right) \pi_{\rho}\left(e_{k l}\right)
$$

for each $\lambda$. From (4.17), we have

$$
\begin{aligned}
& \pi_{\rho}\left(e_{k l}\right) p_{\lambda}^{\rho}\left(e_{i}\right)^{*} p_{\lambda}^{\rho}\left(e_{j}\right)-p_{\lambda}^{\rho}\left(e_{i}\right)^{*} p_{\lambda}^{\rho}\left(e_{j}\right) \pi_{\rho}\left(e_{k l}\right) \\
= & p_{\lambda}^{\rho}\left(\delta_{k i} e_{l}-\delta_{l i} e_{k}\right) p_{\lambda}^{\rho}\left(e_{j}\right)+p_{\lambda}^{\rho}\left(e_{i}\right)^{*} p_{\lambda}^{\rho}\left(\delta_{k j} e_{l}-\delta_{l j} e_{k}\right) .
\end{aligned}
$$

Then it is easy to show that

$$
R_{\rho}(\lambda)=\sum R_{i j k l} p_{\lambda}^{\rho}\left(e_{i}\right)^{*} p_{\lambda}^{\rho}\left(e_{j}\right) \pi_{\rho}\left(e_{k l}\right)=\sum R_{i j k l} \pi_{\rho}\left(e_{k l}\right) p_{\lambda}^{\rho}\left(e_{i}\right)^{*} p_{\lambda}^{\rho}\left(e_{j}\right)=R_{\rho}(\lambda)^{*} .
$$

Since $R_{\rho}^{q}$ and $R_{\rho}^{\mathrm{pf}}$ are linear combinations of $\left\{R_{\rho}(\lambda)\right\}_{\lambda}$ with real coefficients, $R_{\rho}^{q}$ and $R_{\rho}^{\mathrm{pf}}$ are self-adjoint endomorphisms.

We decompose the curvature endomorphisms along (6.3). From (3.10) and (6.2), the Einstein part of $\hat{R}_{\rho}^{q}$ is

$$
\sum_{i j k} \pi_{\rho}\left(\hat{e}_{i j}^{q}\right)\left(E_{i k} \pi_{\rho}\left(e_{k j}\right)-E_{j k} \pi_{\rho}\left(e_{k i}\right)\right)=-\sum_{i j} E_{i j} \pi_{\rho}\left(2 \hat{e}_{i j}^{q+1}+\hat{e}_{i j}^{q}\right) .
$$

Thus $R_{\rho}^{q}$ and $\hat{R}_{\rho}^{q}$ decompose as

$$
\begin{gathered}
R_{\rho}^{q}=\frac{1}{2} \sum_{i j k l} W_{i j k l} \pi_{\rho}\left(e_{i j}^{q} e_{k l}\right)-\sum_{i j} E_{i j} \pi_{\rho}\left(2 e_{i j}^{q+1}+n e_{i j}^{q}\right)+\frac{\pi_{\rho}\left(c_{q+1}\right) \kappa}{n(n-1)}, \\
\hat{R}_{\rho}^{q}=\frac{1}{2} \sum_{i j k l} W_{i j k l} \pi_{\rho}\left(\hat{e}_{i j}^{q} \hat{e}_{k l}\right)-\sum_{i j} E_{i j} \pi_{\rho}\left(2 \hat{e}_{i j}^{q+1}+\hat{e}_{i j}^{q}\right)+\frac{\pi_{\rho}\left(2 \hat{c}_{q+1}-(n-1) \hat{c}_{q}\right) \kappa}{n(n-1)} .
\end{gathered}
$$


Example 6.4. If $M$ is the standard sphere $S^{n}$, then $R_{\rho}^{q}$ is $\pi_{\rho}\left(c_{q+1}\right)$.

We consider $R_{\rho}^{\mathrm{pf}}$. It follows from (4.1), (4.14), and (4.15) that the Einstein part of $R_{\rho}^{\mathrm{pf}}$ is

$$
\sum_{\lambda, i j k} \pi_{\lambda}(\mathrm{pf}) p_{\lambda}^{\rho}\left(e_{i}\right)^{*} p_{\lambda}^{\rho}\left(e_{j}\right)\left(E_{i k} \pi_{\rho}\left(e_{k j}\right)-E_{j k} \pi_{\rho}\left(e_{k i}\right)\right)=0,
$$

and the scalar curvature part is $\pi_{\rho}(\mathrm{pf}) \kappa /(n-1)$.

Proposition 6.5. The curvature endomorphism $R_{\rho}^{\mathrm{pf}}$ does not depend on the Einstein tensor,

$$
R_{\rho}^{\mathrm{pf}}=\frac{1}{2} \sum_{i j k l} W_{i j k l} \pi_{\rho}\left(\mathrm{pf}_{i j} e_{k l}\right)+\frac{\pi_{\rho}(\mathrm{pf}) \kappa}{n-1} .
$$

Corollary 6.6. (1) Suppose that $M$ is an even-dimensional conformally flat manifold; then

$$
R_{\rho}^{\mathrm{pf}}=\frac{\pi_{\rho}(\mathrm{pf}) \kappa}{n-1}
$$

(2) For the exceptional case that $\rho^{m-1}>0$ and $\rho^{m}=0$,

$$
R_{\rho}^{\mathrm{pf}}=\frac{1}{2} \sum_{i j k l} W_{i j k l} \pi_{\rho}\left(\mathrm{pf}_{i j} e_{k l}\right) .
$$

\section{BOCHNER-WEITZENBÖCK FORMULAS}

The second order differential operator $\left(D_{\lambda}^{\rho}\right)^{*} D_{\lambda}^{\rho}$ on $\mathbf{S}_{\rho}$ is realized as

$$
\left(D_{\lambda}^{\rho}\right)^{*} D_{\lambda}^{\rho}=-\sum_{i, j} p_{\lambda}^{\rho}\left(e_{i}\right)^{*} p_{\lambda}^{\rho}\left(e_{j}\right) \nabla_{e_{i}, e_{j}}^{2} .
$$

From (4.10), we have

$$
\sum_{\lambda}\left(D_{\lambda}^{\rho}\right)^{*} D_{\lambda}^{\rho}=-\sum_{\lambda, i, j} p_{\lambda}^{\rho}\left(e_{i}\right)^{*} p_{\lambda}^{\rho}\left(e_{j}\right) \nabla_{e_{i}, e_{j}}^{2}=-\sum_{i, j} \delta_{i j} \nabla_{e_{i}, e_{j}}^{2}=\nabla^{*} \nabla,
$$

where $\nabla^{*} \nabla$ is the connection Laplacian on $\mathbf{S}_{\rho}$ defined by $-\sum_{i} \nabla_{e_{i}, e_{i}}^{2}$.

The universal Bochner-Weitzenböck formula (3.12) gives

$$
\begin{aligned}
\hat{R}_{\rho}^{2 q} & =\sum_{i, j} \pi_{\rho}\left(\hat{e}_{i j}^{2 q}\right)\left(\nabla_{e_{i}, e_{j}}^{2}-\nabla_{e_{j}, e_{i}}^{2}\right) \\
& =-\sum_{\lambda} \hat{w}(\rho ; \lambda)^{2 q}\left(D_{\lambda}^{\rho}\right)^{*} D_{\lambda}^{\rho}-\sum_{i, j} \pi_{\rho}\left(\hat{e}_{j i}^{2 q}+\sum_{p=0}^{2 q-1}(-1)^{p} \hat{c}_{2 q-1-p} \hat{e}_{j i}^{p}\right) \nabla_{e_{j}, e_{i}}^{2} \\
& =\sum_{\lambda}\left\{\sum_{p=0}^{2 q-1} \pi_{\rho}\left(\hat{c}_{2 q-1-p}\right)(-\hat{w}(\rho ; \lambda))^{p}\right\}\left(D_{\lambda}^{\rho}\right)^{*} D_{\lambda}^{\rho} .
\end{aligned}
$$

Similarly (4.14) gives

$$
R_{\rho}^{\mathrm{pf}}=\sum_{\lambda} 2\left(\pi_{\rho}(\mathrm{pf})-\pi_{\lambda}(\mathrm{pf})\right)\left(D_{\lambda}^{\rho}\right)^{*} D_{\lambda}^{\rho} .
$$

We are now in a position to state the main theorem. 
Theorem 7.1 (Bochner-Weitzenböck formulas). Let $\left\{D_{\lambda}^{\rho}\right\}_{\lambda}$ be the gradients on $\mathbf{S}_{\rho}$, and let $\left\{\left(D_{\lambda}^{\rho}\right)^{*}\right\}_{\lambda}$ be their formal adjoints. Then

$$
\begin{gathered}
\sum_{\lambda}\left(D_{\lambda}^{\rho}\right)^{*} D_{\lambda}^{\rho}=\nabla^{*} \nabla \\
\sum_{\lambda}\left\{\sum_{p=0}^{2 q-1} \pi_{\rho}\left(\hat{c}_{2 q-1-p}\right)(-\hat{w}(\rho ; \lambda))^{p}\right\}\left(D_{\lambda}^{\rho}\right)^{*} D_{\lambda}^{\rho}=\hat{R}_{\rho}^{2 q}, \quad q=1,2, \cdots .
\end{gathered}
$$

When $n$ is even, we also have

$$
\sum_{\lambda} 2\left(\pi_{\rho}(\mathrm{pf})-\pi_{\lambda}(\mathrm{pf})\right)\left(D_{\lambda}^{\rho}\right)^{*} D_{\lambda}^{\rho}=R_{\rho}^{\mathrm{pf}}
$$

For the exceptional case, setting $\lambda_{ \pm}:=\rho \pm \mu_{m}$, we have

$$
\left(D_{\lambda_{+}}^{\rho}\right)^{*} D_{\lambda_{+}}^{\rho}-\left(D_{\lambda_{-}}^{\rho}\right)^{*} D_{\lambda_{-}}^{\rho}=-\frac{1}{4 \pi_{\lambda_{+}}(\mathrm{pf})} \sum_{i j k l} W_{i j k l} \pi_{\rho}\left(\mathrm{pf}_{i j} e_{k l}\right) .
$$

Remark 7.2. By using (3.13), we obtain other identities

$$
\hat{R}_{\rho}^{2 q+1}=-\sum_{\lambda}\left\{2 \hat{w}(\rho ; \lambda)^{2 q+1}+\hat{w}(\rho ; \lambda)^{2 q}-\sum_{p=0}^{2 q} \pi_{\rho}\left(\hat{c}_{2 q-p}\right)(-\hat{w}(\rho ; \lambda))^{p}\right\}\left(D_{\lambda}^{\rho}\right)^{*} D_{\lambda}^{\rho}
$$

for $q=0,1, \cdots$. But, from the discussion below, these formulas are linear dependent on (7.2). The above equation for $q=0$ gives a formula by P. Gauduchon [12, Appendix B],

$$
\begin{aligned}
-\frac{1}{2} R_{\rho}^{1} & =-\frac{\pi_{\rho}\left(c_{2}\right) \kappa}{2 n(n-1)}+\sum E_{i k} \pi_{\rho}\left(e_{i k}^{2}\right)-\frac{1}{4} \sum W_{i j k l} \pi_{\rho}\left(e_{i j} e_{k l}\right) \\
& =\sum_{\lambda} w(\rho ; \lambda)\left(D_{\lambda}^{\rho}\right)^{*} D_{\lambda}^{\rho} .
\end{aligned}
$$

Note that this formula can be proved from (4.11).

We shall discuss linear independence of Bochner-Weitzenböck formulas (7.2) and (7.3). We assume that there are $N$ gradients $\left\{D_{\lambda_{i}}^{\rho}\right\}_{i=1}^{N}$ on $\mathbf{S}_{\rho}$. In [3], by using the spectral resolution on the standard sphere, T. Branson shows that there are just $[N / 2]$ independent identities such that $\sum_{i} b_{\lambda_{i}}\left(D_{\lambda_{i}}^{\rho}\right)^{*} D_{\lambda_{i}}^{\rho}$ is a curvature endomorphism. Therefore our task is to prove that (7.2) and (7.3) give $[N / 2]$ independent identities. Define a vector $v(q)$ consisting of the coefficients in (17.2) by

$$
v(q):=\left(\sum_{p=0}^{2 q-1}(-1)^{p} \pi_{\rho}\left(\hat{c}_{2 q-1-p}\right) \hat{w}\left(\rho ; \lambda_{1}\right)^{p}, \cdots, \sum_{p=0}^{2 q-1}(-1)^{p} \pi_{\rho}\left(\hat{c}_{2 q-1-p}\right) \hat{w}\left(\rho ; \lambda_{N}\right)^{p}\right) .
$$


We decompose $(v(1), v(2), \cdots, v(q))$ into the product of a $q \times 2 q$ matrix $C(q)$ and a $2 q \times N$ matrix $W(q)$ given by

$$
\begin{aligned}
& C(q):=\left(\begin{array}{ccccccc}
\pi_{\rho}\left(\hat{c}_{1}\right) & -\pi_{\rho}\left(\hat{c}_{0}\right) & 0 & 0 & \ldots & 0 & 0 \\
\pi_{\rho}\left(\hat{c}_{3}\right) & -\pi_{\rho}\left(\hat{c}_{2}\right) & \pi_{\rho}\left(\hat{c}_{1}\right) & -\pi_{\rho}\left(\hat{c}_{0}\right) & \ldots & 0 & 0 \\
\cdots & \cdots & \cdots & \cdots & \cdots & \ldots & \ldots \\
\pi_{\rho}\left(\hat{c}_{2 q-1}\right) & -\pi_{\rho}\left(\hat{c}_{2 q-2}\right) & \ldots & \cdots & \cdots & \pi_{\rho}\left(\hat{c}_{1}\right) & -\pi_{\rho}\left(\hat{c}_{0}\right)
\end{array}\right), \\
& W(q):=\left(\begin{array}{ccccc}
1 & 1 & \ldots & 1 \\
\hat{w}\left(\rho ; \lambda_{1}\right) & \hat{w}\left(\rho ; \lambda_{2}\right) & \ldots & \hat{w}\left(\rho ; \lambda_{N}\right) \\
\hat{w}\left(\rho ; \lambda_{1}\right)^{2} & \hat{w}\left(\rho ; \lambda_{2}\right)^{2} & \cdots & \hat{w}\left(\rho ; \lambda_{N}\right)^{2} \\
\cdots & \cdots & \cdots & \cdots \\
\hat{w}\left(\rho ; \lambda_{1}\right)^{2 q-1} & \hat{w}\left(\rho ; \lambda_{2}\right)^{2 q-1} & \cdots & \hat{w}\left(\rho ; \lambda_{N}\right)^{2 q-1}
\end{array}\right)
\end{aligned}
$$

Since the conformal weights are different from each other, the rank of the matrix $(v(1), v(2), \cdots, v([N / 2]))=C([N / 2]) W([N / 2])$ is $[N / 2]$ unless it is the exceptional case. For the exceptional case, the rank of $C([N / 2]) W([N / 2])$ is $[N / 2]-1$. But, there is another formula (7.4) independent of (7.2). Thus we have $[N / 2]$ independent Bochner-Weitzenböck formulas.

Corollary 7.3. The formulas (7.2) and (17.3) give all Bochner-Weitzenböck formulas for gradients.

In the rest of this section, we discuss a relation between our Bochner-Weitzenböck formulas and Branson's conformally covariant operators. In [4, Theorem 1.1], Branson classifies the second order conformally covariant operators on associated vector bundles. We consider differential operators,

$$
\begin{gathered}
\left(D_{\lambda_{ \pm}}^{\rho}\right)^{*} D_{\lambda_{ \pm}}^{\rho} \quad \text { for the exceptional case, } \\
\sum_{\lambda} \frac{1}{w(\rho ; \lambda)+\frac{n-2}{2}}\left(D_{\lambda}^{\rho}\right)^{*} D_{\lambda}^{\rho}+\frac{\kappa}{2(n-1)} \text { otherwise. }
\end{gathered}
$$

In the same manner as in Proposition 5.4 we can show that the above operators are conformally covariant. His important observation is that, when $N$ is even, the operator (7.7) has zeroth order. This fact is equivalent to the next proposition.

Proposition 7.4. Assume that $n=2 m$ and $\rho^{m} \neq 0$, or $n=2 m+1$ and $\rho^{m}=1 / 2$. Then, at the symbol level, we have

$$
\sum_{\lambda} \frac{1}{w(\rho ; \lambda)+\frac{n-2}{2}}\left(p_{\lambda}^{\rho}(\xi)^{*} p_{\lambda}^{\rho}(\eta)+p_{\lambda}^{\rho}(\eta)^{*} p_{\lambda}^{\rho}(\xi)\right)=0 \quad \text { for } \xi \text { and } \eta \text { in } \mathbb{R}^{n}
$$

Remark 7.5. Corollary 7.3 implies that we can realize (7.8) as a linear combination of (4.13) for $q=1, \cdots, N / 2$.

For $n=2 m$, we can prove (7.8) directly. We consider the case of $n=2 m$ and $\rho^{m} \neq 0$. Since $\pi_{\rho}(\mathrm{pf})$ is not zero, the equation (7.8) follows from (4.12) and (4.14). Indeed, we can rewrite (7.3) as

$$
\sum_{\lambda} \frac{1}{w(\rho ; \lambda)+\frac{n-2}{2}}\left(D_{\lambda}^{\rho}\right)^{*} D_{\lambda}^{\rho}+\frac{\kappa}{2(n-1)}=-\frac{1}{4 \pi_{\rho}(\mathrm{pf})} \sum W_{i j k l} \pi_{\rho}\left(\mathrm{pf}_{i j} e_{k l}\right) .
$$

Thus the Bochner-Weitzenböck formula associated to the Pfaffian element is just Branson's conformally covariant operator with zeroth order. For the exceptional case, we also have a zeroth order conformally covariant operator, $\left(D_{\lambda_{+}}^{\rho}\right)^{*} D_{\lambda_{+}}^{\rho}-$ $\left(D_{\lambda_{-}}^{\rho}\right)^{*} D_{\lambda_{-}}^{\rho}$ in (17.4). 
For $n=2 m+1$, since there is no Casimir element corresponding to the Pfaffian element, the author does not now have a direct proof of (7.8). Nevertheless, it would be significant to prove that the equation (17.8) provides a Bochner-Weitzenböck formula omitting the Einstein tensor. Let $R_{\rho}(\lambda)$ be the curvature endomorphism given by (6.4). It follows from (7.8) that

$$
\begin{aligned}
\sum_{\lambda} \frac{1}{w(\rho ; \lambda)+\frac{n-2}{2}}\left(D_{\lambda}^{\rho}\right)^{*} D_{\lambda}^{\rho} & =-\frac{1}{2} \sum_{\lambda} \frac{1}{w(\rho ; \lambda)+\frac{n-2}{2}} p_{\lambda}^{\rho}\left(e_{i}\right) p_{\lambda}^{\rho}\left(e_{j}\right)\left(\nabla_{e_{i}, e_{j}}^{2}-\nabla_{e_{j}, e_{i}}^{2}\right) \\
& =-\frac{1}{2} \sum_{\lambda} \frac{1}{w(\rho ; \lambda)+\frac{n-2}{2}} R_{\rho}(\lambda) .
\end{aligned}
$$

Then we shall investigate the curvature endomorphism

$$
\sum_{\lambda} \frac{1}{w(\rho ; \lambda)+\frac{n-2}{2}} R_{\rho}(\lambda) .
$$

It is easy to see that $R_{\rho}(\lambda)$ is equal to

$$
\frac{1}{2} \sum_{i j k l} W_{i j k l} p_{\lambda}^{\rho}\left(e_{i}\right)^{*} p_{\lambda}^{\rho}\left(e_{j}\right) \pi_{\rho}\left(e_{k l}\right)-(2 w(\rho ; \lambda)+n) \sum_{i, j} p_{\lambda}^{\rho}\left(e_{i}\right)^{*} p_{\lambda}^{\rho}\left(e_{j}\right) E_{i j}+\frac{w(\rho ; \lambda) d(\lambda) \kappa}{n(n-1) d(\rho)},
$$

and the Einstein part in (7.10) vanishes because of (6.2) and (7.8). Moreover, we show from (4.21) that the scalar curvature part in (7.10) is $\kappa /(n-1)$. Thus we have

$$
\begin{aligned}
& \sum_{\lambda} \frac{1}{w(\rho ; \lambda)+\frac{n-2}{2}}\left(D_{\lambda}^{\rho}\right)^{*} D_{\lambda}^{\rho}+\frac{\kappa}{2(n-1)} \\
= & -\frac{1}{4} \sum_{\lambda, i, j, k, l} \frac{1}{w(\rho ; \lambda)+\frac{n-2}{2}} W_{i j k l} p_{\lambda}^{\rho}\left(e_{i}\right)^{*} p_{\lambda}^{\rho}\left(e_{j}\right) \pi_{\rho}\left(e_{k l}\right) .
\end{aligned}
$$

Remark 7.6. We know from [4, Theorem 1.1] that, if $N$ is even, up to a constant multiple, there is a unique optimal Bochner-Weitzenböck formula omitting the Einstein tensor: (7.4) for the exceptional case and (7.11) for other cases. If $N$ is odd, there is no such Bochner-Weitzenböck formula.

\section{EXAMPLES}

We give examples and applications of our Bochner-Weitzenböck formulas. We often simply denote $D_{\lambda_{i}}^{\rho}$ by $D_{i}$ in this section.

Example 8.1 (spinors). We discuss gradients on the spinor bundle $\mathbf{S}_{\Delta}$ for $n=$ $2 m+1$. There are two irreducible components in $\mathbf{S}_{\Delta} \otimes T_{\mathbb{C}}(M)$ whose highest weights are $\lambda_{1}=\left(3 / 2,(1 / 2)_{m-1}\right)$ and $\lambda_{2}=\Delta=\left((1 / 2)_{m}\right)$. From (7.1) and (7.5), we have

$$
D_{1}^{*} D_{1}+D_{2}^{*} D_{2}=\nabla^{*} \nabla, \quad \frac{1}{2} D_{1}^{*} D_{1}-\frac{n-1}{2} D_{2}^{*} D_{2}=-\frac{1}{2} R_{\Delta}^{1}=-\frac{\kappa}{8} .
$$

Since the Dirac operator $D$ is $\sqrt{n} D_{2}$ and the twistor operator $T$ is $\sqrt{n /(n-1)} D_{1}$, we have

$$
D^{2}=\nabla^{*} \nabla+\frac{1}{4} \kappa, \quad D^{2}=\frac{n}{4(n-1)} \kappa+T^{*} T .
$$

The first equation gives a vanishing theorem. If $(M, g)$ is a compact spin manifold with positive scalar curvature, then the kernel of $D$ is zero. The second equation gives Friedrich's estimate for eigenvalues of $D^{2}$. Since $T^{*} T$ is a non-negative operator on $M$, each eigenvalue $\mu$ of $D^{2}$ satisfies $\mu \geq \frac{n}{4(n-1)} \min _{x \in M} \kappa(x)$ [11]. 
Example 8.2 (differential forms). We consider the bundle of differential forms $\mathbf{S}_{\left(1_{p}\right)}=\Lambda^{p}(M) \otimes \mathbb{C} \simeq \Lambda^{n-p}(M) \otimes \mathbb{C}$. We have three irreducible components in $\mathbf{S}_{\left(1_{p}\right)} \otimes T_{\mathbb{C}}(M)$ whose highest weights are

$$
\lambda_{1}=\left(2,1_{p-1}\right), \quad \lambda_{2}=\left(1_{p+1}\right), \quad \lambda_{3}=\left(1_{p-1}\right) .
$$

From (7.1) and (7.5), we get

$$
\begin{gathered}
D_{1}^{*} D_{1}+D_{2}^{*} D_{2}+D_{3}^{*} D_{3}=\nabla^{*} \nabla \\
D_{1}^{*} D_{1}-p D_{2}^{*} D_{2}-(n-p) D_{3}^{*} D_{3}=-\frac{1}{2} R_{\left(1_{p}\right)}^{1} .
\end{gathered}
$$

The operators $D_{1}, D_{2}$ and $D_{3}$ are constant multiples of the conformal Killing operator $C$, the exterior derivative $d$ and the interior derivative $d^{*}$, respectively. We normalize $\left\{D_{i}\right\}_{1 \leq i \leq 3}$ and obtain

$$
\begin{gathered}
C^{*} C+\frac{1}{p+1} d^{*} d+\frac{1}{n-p+1} d d^{*}=\nabla^{*} \nabla, \\
C^{*} C-\frac{p}{p+1} d^{*} d-\frac{n-p}{n-p+1} d d^{*}=-\frac{1}{2} R_{\left(1_{p}\right)}^{1} .
\end{gathered}
$$

In particular, we get a well-known formula, $d^{*} d+d^{*} d=\nabla^{*} \nabla+R_{\left(1_{p}\right)}^{1} / 2$.

By using (8.1), we can prove eigenvalue estimates of the Laplace operator $d^{*} d+$ $d d^{*}$, Lichnerowicz's estimate for functions and Gallot-Meyer's estimate for differential forms [13. Suppose that $(M, g)$ is a compact Riemannian manifold. For eigenfunction $f$ of $d^{*} d$ with non-zero eigenvalue $\mu$, we have

$$
\begin{aligned}
\mu\|d f\|^{2} & =\left(d d^{*} d f, d f\right)=\frac{n}{n-1}\left(\left(C^{*} C+\frac{1}{2} R_{\Lambda^{1}}^{1}\right) d f, d f\right) \\
& =\frac{n}{n-1}\left\{\|C d f\|^{2}+(\operatorname{Ric}(d f), d f)\right\} \geq \frac{n}{n-1}(\operatorname{Ric}(d f), d f),
\end{aligned}
$$

where $(\phi, \psi)$ denotes $\int_{M}\langle\phi, \psi\rangle d v$. Accordingly, non-zero eigenvalue of $d^{*} d$ on $\Lambda^{0}(M)$ has a lower bound depending on the Ricci curvature.

Suppose that $(M, g)$ is a compact Riemannian manifold of positive curvature. In other words, there exists a constant $r>0$ such that $R_{i j k l} \geq r\left(\delta_{i l} \delta_{j k}-\delta_{i k} \delta_{j l}\right)$. For $\phi$ in $\Gamma\left(M, \Lambda^{p}(M)\right)$,

$$
\begin{aligned}
\left(\left(d^{*} d+d^{*} d\right) \phi, \phi\right) & \geq \frac{n-p+1}{n-p}\left(\left(\frac{p}{p+1} d^{*} d+\frac{n-p}{n-p+1} d d^{*}\right) \phi, \phi\right) \\
& =\frac{n-p+1}{n-p}\|C \phi\|^{2}+\frac{n-p+1}{2(n-p)}\left(R_{\left(1_{p}\right)}^{1} \phi, \phi\right) \\
& \geq \frac{n-p+1}{2(n-p)} r \pi_{\left(1_{p}\right)}\left(c_{2}\right)\|\phi\|^{2}=p(n-p+1) r\|\phi\|^{2} .
\end{aligned}
$$

Therefore the eigenvalue $\mu$ of $d^{*} d+d d^{*}$ on $\Lambda^{p}(M)$ satisfies $\mu \geq p(n-p+1) r$.

Example 8.3 (The conformal Weyl tensor). Let $(M, g)$ be an $n$-dimensional Riemannian manifold with $n \geq 5$. The four-dimensional case is discussed in the next section. The conformal Weyl tensor $W=W_{i j k l}$ is a section of $\mathbf{S}_{\rho}$ with $\rho=\left(2_{2}\right)$. The highest weights of irreducible components in $\mathbf{S}_{\rho} \otimes T_{\mathbb{C}}(M)$ are

$$
\left\{\begin{array}{llll}
(3,2), & \left(2_{2}\right), \quad(2,1), & n=5 \\
(3,2), & \left(2_{2}, 1\right), \quad\left(2_{2},-1\right), & (2,1), & n=6 \\
(3,2) & \left(2_{2}, 1\right), \quad(2,1), & n \geq 7 .
\end{array}\right.
$$


Because of the second Bianchi identity, the projections of $\nabla W$ to $\mathbf{S}_{\left(2_{2}, \pm 1\right)}$ and $\mathbf{S}_{\left(2_{2}\right)}$ are zero 23. Setting $\lambda_{1}:=(3,2)$ and $\lambda_{2}:=(2,1)$, we show from (7.1) and (7.5) that there is a Bochner-Weitzenböck formula for the conformal Weyl tensor,

$$
\frac{n+1}{2} D_{2}^{*} D_{2} W=\nabla^{*} \nabla W+\frac{1}{4} R_{\rho}^{1} W .
$$

It is known that $D_{2} W$ is a constant multiple of $\sum_{s}\left(\nabla^{s} W_{s i j k}+\nabla^{s} W_{s j i k}\right)$ [23]. Then $D_{2} W=0$ is equivalent to $\delta W=0$, where $\delta W:=-\sum \nabla^{s} W_{s i j k}$. If $(M, g)$ is a Riemannian manifold with $\delta W=0$, then $W$ satisfies

$$
\nabla^{*} \nabla W+\frac{1}{4} R_{\rho}^{1} W=0
$$

Example 8.4 (The exceptional case). We consider the exceptional case that $n=$ $2 m$ and $\rho^{m-1}>\rho^{m}=0$. When $\rho$ is $\left(1_{m-1}\right), D_{\lambda_{ \pm}}^{\rho}$ is a constant multiple of $d_{ \pm}$ on $\Lambda^{m-1}(M) \otimes \mathbb{C}$, where $d_{ \pm}$denotes $\frac{1}{2}(1 \pm *) d$ for $m=2 l$ and $\frac{1}{2}(1 \pm \sqrt{-1} *) d$ for $m=2 l+1$. Then we have $d_{+}^{*} d_{+}=d_{-}^{*} d_{-}$. But, in general, $\left(D_{\lambda_{+}}^{\rho}\right)^{*} D_{\lambda_{+}}^{\rho}$ is not equal to $\left(D_{\lambda_{-}}^{\rho}\right)^{*} D_{\lambda_{-}}^{\rho}$. If $(M, g)$ is a conformally flat manifold, then we have $\left(D_{\lambda_{+}}^{\rho}\right)^{*} D_{\lambda_{+}}^{\rho}=\left(D_{\lambda_{-}}^{\rho}\right)^{*} D_{\lambda_{-}}^{\rho}$ from (7.4).

Example 8.5 (Branson-Hijazi vanishing theorem). We consider the case of $n=2 m$ and $\rho=\left(p_{m-1}, \pm p\right)$ for $p=1,3 / 2,2,5 / 2, \cdots$. We have two irreducible components in $\mathbf{S}_{\rho} \otimes T_{\mathbb{C}}(M)$ and set $\lambda_{1}:=\left(p+1, p_{m-2}, \pm p\right)$ and $\lambda_{2}:=\left(p_{m-1}, \pm p \mp 1\right)$. Then we have

$$
\begin{gathered}
D_{1}^{*} D_{1}+D_{2}^{*} D_{2}=\nabla^{*} \nabla \\
p D_{1}^{*} D_{1}+(-p-m+1) D_{2}^{*} D_{2}=-\frac{1}{2} R_{\rho}^{1}, \\
2\left(\pi_{\rho}(\mathrm{pf})-\pi_{\lambda_{1}}(\mathrm{pf})\right) D_{1}^{*} D_{1}+2\left(\pi_{\rho}(\mathrm{pf})-\pi_{\lambda_{2}}(\mathrm{pf})\right) D_{2}^{*} D_{2}=R_{\rho}^{\mathrm{pf}} .
\end{gathered}
$$

The second identity is linear dependent on the third one. We actually have $\pi_{\rho}(\mathrm{pf}) R_{\rho}^{1}$ $=p(p+m-1) R_{\rho}^{\mathrm{pf}}$. Since $R_{\rho}^{\mathrm{pf}}$ omits the Einstein tensor, we have

$$
\frac{2 p+m-1}{p} D_{2}^{*} D_{2}=\nabla^{*} \nabla+\frac{p+m-1}{4 m-2} \kappa+\frac{1}{4 p} \sum W_{i j k l} \pi_{\rho}\left(e_{i j} e_{k l}\right) .
$$

T. Branson and O. Hijazi give a vanishing theorem as follows [7, Theorem 4.2]. We diagonalize $W_{i j k l}$ such that $W_{i j k l}=c_{i j}(x)\left(\delta_{i l} \delta_{j k}-\delta_{i k} \delta_{j l}\right)$ on each point $x$ in $M$. Then

$$
\pi_{\rho}\left(c_{2}\right) \min _{i<j} c_{i j}(x) \leq \frac{1}{2} \sum_{i j k l} W_{i j k l} \pi_{\rho}\left(e_{i j} e_{k l}\right)=\sum_{i j} c_{i j} \pi_{\rho}\left(e_{i j} e_{j i}\right) \leq \pi_{\rho}\left(c_{2}\right) \max _{i<j} c_{i j}(x) .
$$

Supposing $\frac{\kappa(x)}{n(n-1)}+\min c_{i j}(x)>0$ for all $x$ in M, we have $\operatorname{ker} D_{2}=0$ by (8.2). In particular, for $\rho=\left(1_{m-1}, \pm 1\right)$, we have Bourguignon's vanishing theorem, $H^{m}(M, \mathbb{R})=0$ in 2 . They also discuss a relation to the first eigenvalue of Yamabe Laplacian in [6], 7].

Through Examples 8.2 and 8.5 we note that it needs to investigate curvature endomorphisms for vanishing theorems and eigenvalue estimates. We diagonalize $R_{i j k l}$ such that $R_{i j k l}=r_{i j}(x)\left(\delta_{i l} \delta_{j k}-\delta_{i k} \delta_{j l}\right)$. Then we have $\hat{R}_{\rho}^{q}=\sum_{i j} r_{i j} \pi_{\rho}\left(\hat{e}_{i j}^{q} \hat{e}_{j i}\right)$. 
The curvature endomorphism $\hat{R}_{\rho}^{q}$ for $q=1,2$ can be estimated,

$$
\pi_{\rho}\left(c_{2}\right) \min _{i<j} r_{i j}(x) \leq \hat{R}_{\rho}^{1}=\frac{2}{n} \hat{R}_{\rho}^{2}=\sum_{i j} r_{i j} \pi_{\rho}\left(e_{i j} e_{j i}\right) \leq \pi_{\rho}\left(c_{2}\right) \max _{i<j} r_{i j}(x) .
$$

Since we do not know whether $\pi_{\rho}\left(\hat{e}_{i j}^{q} \hat{e}_{j i}\right)$ for $q \geq 3$ is non-negative, it is difficult to estimate $\hat{R}_{\rho}^{q}$ for $q \geq 3$. But, in the 4 -dimensional case, we can estimate the curvature endomorphisms more precisely using the decomposition $\Lambda^{2}(M)=\Lambda_{+}^{2}(M) \oplus \Lambda_{-}^{2}(M)$.

Remark 8.6. It is possible to estimate the Einstein part and the scalar curvature part of curvature endomorphisms. For example, the Einstein part of $R_{\rho}^{3}-n R_{\rho}^{2} / 2+$ $n^{2} R_{\rho}^{1} / 4$ is $-\sum_{i j} 2 \pi_{\rho}\left(e_{i j}^{4}\right) E_{i j}$, which we can estimate because of $\pi_{\rho}\left(e_{i j}^{4}\right) \geq 0$.

\section{The FOUR-DIMENSIONAL CASE}

In this section, we discuss the four-dimensional case. We begin with basic facts of four-dimensional geometry. The Hodge star operator $*$ decomposes $\Lambda^{2}\left(\mathbb{R}^{4}\right)$ into the self-dual part $\Lambda_{+}^{2}$ and the anti-self-dual part $\Lambda_{-}^{2}$. For an oriented orthonormal basis $e=\left(e_{1}, e_{2}, e_{3}, e_{4}\right)$ of $\mathbb{R}^{4}$, we set a basis of $\Lambda_{ \pm}^{2}$ by

$$
X_{1}^{ \pm}:=\frac{1}{2}\left(e_{14} \pm e_{23}\right), \quad X_{2}^{ \pm}:=-\frac{1}{2}\left(e_{13} \pm e_{42}\right), \quad X_{3}^{ \pm}:=\frac{1}{2}\left(e_{12} \pm e_{34}\right) .
$$

Identifying $\Lambda^{2}\left(\mathbb{R}^{4}\right)$ with $\mathfrak{s o}(4)=\mathfrak{s o}(3) \oplus \mathfrak{s o}(3)$, we get

$$
\left[X_{i}^{ \pm}, X_{j}^{ \pm}\right]=\sum_{1 \leq k \leq 3} \epsilon_{i j k} X_{k}^{ \pm}, \quad\left[X_{i}^{+}, X_{j}^{-}\right]=0
$$

where

$$
\epsilon_{i j k}= \begin{cases}\operatorname{sgn}\left(\begin{array}{lll}
1 & 2 & 3 \\
i & j & k
\end{array}\right), & \{i, j, k\}=\{1,2,3\} \\
0, & \text { otherwise. }\end{cases}
$$

Thus $\left\{X_{i}^{ \pm}\right\}_{i}$ is a standard basis of $\mathfrak{s o}(3)$,

$$
X_{1}^{ \pm}=\left(\begin{array}{ccc}
0 & 0 & 0 \\
0 & 0 & -1 \\
0 & 1 & 0
\end{array}\right), \quad X_{2}^{ \pm}=\left(\begin{array}{ccc}
0 & 0 & 1 \\
0 & 0 & 0 \\
-1 & 0 & 0
\end{array}\right), \quad X_{3}^{ \pm}=\left(\begin{array}{ccc}
0 & -1 & 0 \\
1 & 0 & 0 \\
0 & 0 & 0
\end{array}\right) \text {. }
$$

Let $(M, g)$ be a four-dimensional oriented Riemannian or spin manifold. We consider the Riemannian curvature $R_{T}$ as an endomorphism of $\Lambda^{2}(M)$,

$$
R_{T}: \Lambda^{2}(M) \ni e_{i j} \mapsto \frac{1}{2} \sum R_{i j k l} e_{k l} \in \Lambda^{2}(M) .
$$

Then we realize $R_{T}$ as a $6 \times 6$ matrix with respect to basis $\left\{X_{i}^{+}\right\}_{i} \cup\left\{X_{j}^{-}\right\}_{j}$,

$$
\left(\begin{array}{cc}
W^{+} & 0 \\
0 & W^{-}
\end{array}\right)+\left(\begin{array}{cc}
0 & K \\
K^{t} & 0
\end{array}\right)+\left(\begin{array}{cc}
-\kappa / 12 & 0 \\
0 & -\kappa / 12
\end{array}\right),
$$

where $W^{+}$(resp. $W^{-}$) is the self-dual (resp. anti-self-dual) conformal Weyl tensor and $K$ corresponds to $K_{i j k l}$ in (6.1). In other words, we have

$$
\begin{aligned}
& R_{T}\left(X_{i}^{+}\right)=\sum W_{i j}^{+} X_{j}^{+}+\sum K_{j i} X_{j}^{-}-\frac{\kappa}{12} X_{i}^{+}, \\
& R_{T}\left(X_{i}^{-}\right)=\sum W_{i j}^{-} X_{j}^{-}+\sum K_{i j} X_{j}^{+}-\frac{\kappa}{12} X_{i}^{-} .
\end{aligned}
$$


From now on, we denote the highest weight $\rho$ of $\mathfrak{s o}(4)$ by $\rho=\left(\frac{k+l}{2}, \frac{k-l}{2}\right)$ for nonnegative integers $k$ and $l$. Note that $\rho$ corresponds to the highest weight $(k / 2) \hat{\otimes}(l / 2)$ of $\mathfrak{s o}(3) \oplus \mathfrak{s o}(3)$.

We define a curvature endomorphism on $\mathbf{S}_{\rho}$ by

$$
R_{\rho}^{ \pm}:=4 \sum_{i} \pi_{\rho}\left(X_{i}^{ \pm} R_{T}\left(X_{i}^{ \pm}\right)\right) .
$$

Since $4 \sum X_{i}^{+} X_{i}^{+}=-c_{2} / 2-2$ pf and $4 \sum X_{i}^{-} X_{i}^{-}=-c_{2} / 2+2$ pf, we have

$$
\begin{aligned}
& R_{\rho}^{+}=4 \sum_{i j} W_{i j}^{+} \pi_{\rho}\left(X_{i}^{+} X_{j}^{+}\right)+4 \sum_{i j} K_{i j} \pi_{\rho}\left(X_{i}^{-} X_{j}^{+}\right)+\frac{k(k+2)}{12} \kappa, \\
& R_{\rho}^{-}=4 \sum_{i j} W_{i j}^{-} \pi_{\rho}\left(X_{i}^{-} X_{j}^{-}\right)+4 \sum_{i j} K_{i j} \pi_{\rho}\left(X_{i}^{-} X_{j}^{+}\right)+\frac{l(l+2)}{12} \kappa .
\end{aligned}
$$

Lemma 9.1. (1) The curvature endomorphisms $R_{\rho}^{1}$ and $R_{\rho}^{\mathrm{pf}}$ satisfy

$$
R_{\rho}^{1}=R_{\rho}^{+}+R_{\rho}^{-}, \quad R_{\rho}^{\mathrm{pf}}=R_{\rho}^{+}-R_{\rho}^{-} .
$$

(2) If $\rho$ is $(k / 2, k / 2)$, then $R_{\rho}^{-}$is zero and $R_{\rho}^{+}$does not depend on the Einstein tensor. If $\rho$ is $\left(\frac{k+1}{2}, \frac{k-1}{2}\right)$, then $R_{\rho}^{-}$does not depend on $W^{-}$.

Proof. By using (4.5), we can show (9.2) straightforwardly. We shall prove the second claim. When $\rho$ is $(k / 2, k / 2), \pi_{\rho}\left(X_{i}^{-}\right)$is zero for each $i$. Then $R_{\rho}^{-}$is zero and $R_{\rho}^{+}$ does not depend on the Einstein tensor. When $\rho$ is $\left(\frac{k+1}{2}, \frac{k-1}{2}\right),\left\{\pi_{\rho}\left(X_{i}^{-}\right)\right\}_{1 \leq i \leq 3}$ gives the spin $1 / 2$ representation of $\mathfrak{s o}(3)=\mathfrak{s u}(2)$. Then we have $\sum_{i j} W_{i j}^{-} \pi_{\rho}\left(X_{i}^{-} X_{j}^{-}\right)=0$ because of $W_{i j}^{-}=W_{j i}^{-}$and $\sum W_{i i}^{-}=0$.

The irreducible decomposition of $\mathbf{S}_{\rho} \otimes T_{\mathbb{C}}(M)$ is $\bigoplus_{i=1}^{4} \mathbf{S}_{\lambda_{i}}$, where

$$
\lambda_{1}=\rho+\mu_{1}, \quad \lambda_{2}=\rho+\mu_{2}, \quad \lambda_{3}=\rho-\mu_{2}, \quad \lambda_{4}=\rho-\mu_{1} .
$$

From (7.1), (7.2) and (7.3), we have all Bochner-Weitzenböck formulas for the fourdimensional case,

$$
\begin{aligned}
D_{1}^{*} D_{1}+D_{2}^{*} D_{2}+D_{3}^{*} D_{3}+D_{4}^{*} D_{4} & =\nabla^{*} \nabla, \\
k D_{1}^{*} D_{1}+k D_{2}^{*} D_{2}-(k+2) D_{3}^{*} D_{3}-(k+2) D_{4}^{*} D_{4} & =-R_{\rho}^{+}, \\
l D_{1}^{*} D_{1}-(l+2) D_{2}^{*} D_{2}+l D_{3}^{*} D_{3}-(l+2) D_{4}^{*} D_{4} & =-R_{\rho}^{-} .
\end{aligned}
$$

We shall state some vanishing theorems. First, we consider the case that $\rho$ is $(k / 2, k / 2)$ for a positive integer $k$. Then we get $D_{2}=D_{4}=0$ and

$$
\frac{2(k+1)}{k} D_{3}^{*} D_{3}=\nabla^{*} \nabla+\frac{4}{k} \sum W_{i j}^{+} \pi_{\rho}\left(X_{i}^{+} X_{j}^{+}\right)+\frac{k+2}{12} \kappa .
$$

Example 9.2. If $M$ is a compact anti-self-dual manifold, then

$$
\frac{2(k+1)}{k} D_{3}^{*} D_{3}=\nabla^{*} \nabla+\frac{k+2}{12} \kappa .
$$

The kernel of $D_{3}$ is isomorphic to a cohomology on the twistor space of $M$ [14].

Example 9.3 (self-dual 2-forms). For $\rho=(1,1)$, the associated vector bundle $\mathbf{S}_{\rho}$ is $\Lambda_{+}^{2}(M) \otimes \mathbb{C}$ and the kernel of $D_{3}$ is the space of harmonic self-dual 2-forms. If $\omega$ 
is in $\operatorname{ker} D_{3}$, then we have a formula in [2],

$$
0=\left\langle\nabla^{*} \nabla \omega, \omega\right\rangle+2\left\langle W^{+}(\omega), \omega\right\rangle+\frac{1}{3} \kappa\langle\omega, \omega\rangle,
$$

where $W^{+}(\omega)=W^{+}\left(\sum \omega_{i} X_{i}^{+}\right)=\sum \omega_{i} W_{i j}^{+} X_{j}^{+}$.

Example 9.4 (the self-dual conformal Weyl tensor). For $\rho=(2,2)$, the vector bundle $\mathbf{S}_{\rho}$ is embedded into $\Lambda_{+}^{2}(M) \otimes\left(\Lambda_{+}^{2}(M)\right)^{*} \otimes \mathbb{C}$ and the sections of $\mathbf{S}_{\rho}$ are realized locally as trace-free symmetric $3 \times 3$ matrices. Therefore we have $\langle Z, Z\rangle=$ $\operatorname{tr}(Z \bar{Z})$ and $\pi_{\rho}\left(X_{i}^{+}\right) Z=X_{i}^{+} Z-Z X_{i}^{+}$for $Z$ in $\Gamma\left(M, \mathbf{S}_{\rho}\right)$. Here we use the matrix realization (9.1) of $\left\{X_{i}^{+}\right\}_{i}$. It follows that

$$
\begin{aligned}
\sum W_{i j}^{+} \pi_{\rho}\left(X_{i}^{+} X_{j}^{+}\right) Z & =\sum W_{i j}^{+}\left(X_{j}^{+} X_{i}^{+} Z-X_{j}^{+} Z X_{i}^{+}-X_{i}^{+} Z X_{j}^{+}+Z X_{i}^{+} X_{j}^{+}\right) \\
& =W^{+} Z+Z W^{+}-2 \sum W_{i j}^{+} X_{i}^{+} Z X_{j}^{+} .
\end{aligned}
$$

Choosing $W^{+}$as a section $Z$ of $\mathbf{S}_{\rho}$, we have

$$
\begin{aligned}
\left\langle\sum W_{i j}^{+} \pi_{\rho}\left(X_{i}^{+} X_{j}\right) W^{+}, W^{+}\right\rangle & =2 \operatorname{tr}\left(\left(W^{+}\right)^{3}\right)+4 \operatorname{tr}\left(\left(W^{+}\right)^{3}\right)=6 \operatorname{tr}\left(\left(W^{+}\right)^{3}\right) \\
& =18 \operatorname{det}\left(W^{+}\right) .
\end{aligned}
$$

If $\delta W^{+}=-\sum_{s} \nabla^{s} W_{s i j k}^{+}=0$, then $D_{3} W^{+}$is zero and $W^{+}$satisfies the following equation in [1, pp. 451-455]:

$$
0=\left\langle\nabla^{*} \nabla W^{+}, W^{+}\right\rangle+6 \operatorname{tr}\left(\left(W^{+}\right)^{3}\right)+\frac{\kappa}{2} \operatorname{tr}\left(\left(W^{+}\right)^{2}\right) .
$$

Next we consider the case that the highest weight $\rho$ is $\left(\frac{k+1}{2}, \frac{k-1}{2}\right)$ for a positive integer $k$. Then we have

$$
\begin{array}{r}
\nabla^{*} \nabla-1 / 3 R_{\rho}^{-}=4 / 3\left(D_{1}^{*} D_{1}+D_{3}^{*} D_{3}\right), \\
\nabla^{*} \nabla+R_{\rho}^{-}=4\left(D_{2}^{*} D_{2}+D_{4}^{*} D_{4}\right),
\end{array}
$$

where

$$
R_{\rho}^{-}=4 \sum K_{i j} \pi_{\rho}\left(X_{i}^{-} X_{j}^{+}\right)+\kappa / 4
$$

Example 9.5. If $(M, g)$ is a compact Einstein manifold with positive (resp. negative) constant scalar curvature, then $\operatorname{ker} D_{2} \cap \operatorname{ker} D_{4}\left(\right.$ resp. $\left.\operatorname{ker} D_{1} \cap \operatorname{ker} D_{3}\right)$ is zero.

\section{ACKNowledgement}

The author thanks David M. J. Calderbank and the referees for good advice and suggestions.

\section{REFERENCES}

1. A. Besse, Einstein manifolds, Springer-Verlag, Berlin, Heidelberg, 1987. MR0867684 (88f:53087)

2. J.P. Bourguignon, Les variétés de dimension 4 à signature non nulle dont la courbure est harmonique sont d'Einstein, Invent. Math. 63 (1981), 263-286. MR0610539 (82g:53051)

3. T. Branson, Stein-Weiss operators and ellipticity, J. Funct. Anal. 151 (1997), 334-383. MR 1491546 (99b:58219)

4. T. Branson, Second order conformal covariants, Proc. Amer. Math. Soc. 126 (1998), 10311042. MR:1422849 (98f:53012) 
5. T. Branson, Kato constants in Riemannian geometry, Math. Res. Lett. 7 (2000), 245-261. MR.1764320 (2001i:58066)

6. T. Branson and O. Hijazi, Vanishing theorems and eigenvalue estimates in Riemannian spin geometry, Internat. J. Math. 8 (1997), 921-934. MR.1482970 (98k:58229)

7. T. Branson and O. Hijazi, Improved forms of some vanishing theorems in Riemannian spin geometry, Internat. J. Math. 11 (2000), 291-304. MR1769610 (2001d:53056)

8. T. Branson and O. Hijazi, Bochner-Weitzenböck formulas associated with the RaritaSchwinger operator, Internat. J. Math. 13 (2002), no. 2, 137-182. MR1891206 (2003c:53063)

9. D. Calderbank, P. Gauduchon and M. Herzlich, Refined Kato inequalities and conformal weights in Riemannian geometry, J. Funct. Anal. 173 (2000), 214-255. MR1760284 (2001f:58046)

10. H. D. Fegan, Conformally invariant first order differential operators, Quart. J. Math. Oxford, 27 (1976), 371-378. MR0482879 (58:2920)

11. T. Friedrich, Dirac operators in Riemannian Geometry, Graduate Studies in Math. 25. American Mathematical Society, Providence, RI, 2000. MR.1777332 (2001c:58017)

12. P. Gauduchon, Structures de Weyl et théorèmes d'annulation sur une variété conforme autoduale, Ann. Scuola Norm. Sup. Pisa Cl. Sci. (4) 18 (1991), 563-629. MR.1153706 (93d:32046)

13. S. Gallot and D. Meyer, Opérateur de courbure et laplacien des formes différentielles d'une variété Rimannienne, J. Math. Pures Appl. (9) 54 (1975) 259-284. MR0454884 (56:13128)

14. N. J. Hitchin, Linear field equations on self-dual spaces, Proc. Roy. Soc. London, A 370 (1980), 173-191. MR0563832 (81i:81057)

15. Y. Homma, Bochner identities for Kählerian gradients, to appear in Math. Ann.

16. Y. Homma, Casimir elements and Bochner identities on Riemannian manifolds, Prog. in Math. Phys. 34 (2003), Birkhäuser, 185-200. MR2025980 (2004m:58055)

17. Y. Homma, Universal Bochner-Weitzenböck formulas for hyper-Kählerian gradients, in "Advances in Analysis and Geometry", Trend in Math., Birkhäuser (2004), 189-208. MR2077086 (2005f:53066)

18. A. W. Knapp, Representation Theory of Semisimple Groups, Princeton Math. Series, 36. Princeton University Press, Princeton, NJ, 1986. MR.0855239 (87j:22022)

19. H. B. Lawson and M. L. Michelsohn, Spin geometry, Princeton Math. Series, 38. Princeton University Press, Princeton, NJ, 1989. MR1031992 (91g:53001)

20. C. O. Nwachuku and M. A. Rashid, Eigenvalues of the Casimir operators of the orthogonal and symplectic groups, J. Math. Phys. 17 (1976), no. 8, 1611-1616. MR0411409 (53:15145)

21. S. Okubo, Casimir invariants and vector operators in simple and classical Lie algebras, J. Math. Phys. 18 (1977), 2382-2394.

22. E. M. Stein and G. Weiss, Generalization of the Cauchy-Riemann equations and representation of the rotation group, Amer. J. Math. 90 (1968), 163-196. MR0223492 (36:6540)

23. R. Strichartz, Linear algebra of curvature tensors and their covariant derivatives, Canad. J. Math. 40 (1988), 1105-1143. MR0973512 (90c:53048)

24. D. P. Želobenko, Compact Lie Groups and Their Representations, Translations of Mathematical. Monographs, 40. American Mathematical Society, Providence, RI, 1973. MR0473098 $(57: 12776 \mathrm{~b})$

Department of Mathematics, Faculty of Science and Technology, Science University of Tokyo, 2641 Noda, Chiba, 278-8510, JAPAN

E-mail address: homma_yasushi@ma.noda.tus.ac.jp 